\title{
STRICHARTZ ESTIMATES FOR SCHRÖDINGER EQUATIONS ON IRRATIONAL TORI
}

\author{
ZIHUA GUO, TADAHIRO OH, AND YUZHAO WANG \\ Dedicated to the memory of Professor Harold N. Shapiro (1922-2013)
}

\begin{abstract}
In this paper, we prove new Strichartz estimates for linear Schrödinger equations posed on $d$-dimensional irrational tori. Then, we use these estimates to prove subcritical and critical local well-posedness results for nonlinear Schrödinger equations (NLS) on irrational tori.
\end{abstract}

\section{InTRODUCTION}

1.1. Background. The Cauchy problem of the nonlinear Schrödinger equation (NLS):

$$
\left\{\begin{array}{l}
i \partial_{t} u-\Delta u= \pm|u|^{p-1} u \\
\left.u\right|_{t=0}=u_{0} \in H^{s}(M),
\end{array} \quad(\mathbf{x}, t) \in M \times \mathbb{R}\right.
$$

has been studied extensively in different settings (for example, $M=\mathbb{R}^{d}, \mathbb{T}^{d}$, and certain classes of manifolds) over recent years [16, 39, 28, 13, 3, 4, 9, 10, 30, 21, 22, 20, 42]. See also the following monographs [36, 12, 38] for references therein. In the study of NLS (1.1), Strichartz estimates of the following type have played a fundamental role

$$
\left\|e^{-i t \Delta} f\right\|_{L_{t}^{q} L_{\mathbf{x}}^{r}(\mathbb{R} \times M)} \lesssim\|f\|_{H_{\mathbf{x}}^{s}(M)},
$$

where $\|f\|_{L_{t}^{q} L_{\mathbf{x}}^{r}}=\|\| f(\mathbf{x}, t)\left\|_{L_{\mathbf{x}}^{r}}\right\|_{L_{t}^{q}}$ 五 In particular, when $M=\mathbb{R}^{d}$, (1.2) is known to hold with $s=0$, namely

$$
\left\|e^{-i t \Delta} f\right\|_{L_{t}^{q} L_{\mathbf{x}}^{r}\left(\mathbb{R} \times \mathbb{R}^{d}\right)} \lesssim\|f\|_{L_{\mathbf{x}}^{2}\left(\mathbb{R}^{d}\right)}
$$

if and only if $(q, r)$ satisfies $\frac{2}{q}+\frac{d}{r}=\frac{d}{2}$ with $2 \leq q, r \leq \infty$ and $(q, r, d) \neq(2, \infty, 2)$. See [34, 44, 17, 29]. This was first obtained for the case $q=r$ by Strichartz [34] via the Fourier restriction method. It was then generalized by a combination of the duality argument and the following dispersive estimate:

$$
\left\|e^{-i t \Delta} f\right\|_{L_{\mathbf{x}}^{\infty}\left(\mathbb{R}^{d}\right)} \lesssim|t|^{-\frac{d}{2}}\|f\|_{L_{\mathbf{x}}^{1}\left(\mathbb{R}^{d}\right)} .
$$

The endpoint case $(q, r)=\left(2, \frac{2 d}{d-2}\right), d \neq 2$, was then proven in [29].

2010 Mathematics Subject Classification. 35Q55, $42 \mathrm{~B} 37$.

Key words and phrases. nonlinear Schrödinger equation; irrational torus; Strichartz estimate; wellposedness.

${ }^{1}$ We use $A \lesssim B$ to denote an estimate of the form $A \leq C B$ for some $C>0$. Similarly, we use $A \sim B$ to denote $A \lesssim B$ and $B \lesssim A$ 
Now, consider $f \in L^{2}\left(\mathbb{R}^{d}\right)$ with supp $\widehat{f} \subset[-N, N]^{d}$. Then, as an immediate corollary to (1.3), we have the following Strichartz estimate on $\mathbb{R}^{d}$ :

$$
\left\|e^{-i t \Delta} f\right\|_{L_{t, \mathbf{x}}^{p}\left(\mathbb{R} \times \mathbb{R}^{d}\right)} \lesssim N^{\frac{d}{2}-\frac{d+2}{p}}\|f\|_{L^{2}\left(\mathbb{R}^{d}\right)},
$$

for $\frac{2(d+2)}{d} \leq p \leq \infty$ on $\mathbb{R}^{d}$. Indeed, on the one hand, the Strichartz estimate (1.3) with $q=r=\frac{2(d+2)}{d}$ gives

$$
\left\|e^{-i t \Delta} f\right\|_{L_{t, \mathbf{x}}{ }^{\frac{2(d+2)}{d}}\left(\mathbb{R} \times \mathbb{R}^{d}\right)} \lesssim\|f\|_{L^{2}\left(\mathbb{R}^{d}\right)} .
$$

On the other hand, by Bernstein's inequality [43, Chapter 5], we have

$$
\left\|e^{-i t \Delta} f\right\|_{L_{t, \mathbf{x}}^{\infty}\left(\mathbb{R} \times \mathbb{R}^{d}\right)} \lesssim N^{\frac{d}{2}}\|f\|_{L^{2}\left(\mathbb{R}^{d}\right)}
$$

for all $f \in L^{2}\left(\mathbb{R}^{d}\right)$ with supp $\widehat{f} \subset[-N, N]^{d}$. By interpolating (1.6) and (1.7), we see that the estimate (1.5) holds for $\frac{2(d+2)}{d} \leq p \leq \infty$ and is sharp in view of sharpness of (1.6) and (1.7). Note that the estimate (1.5) is scaling-invariant in the following sense. Consider the linear Schrödinger equation:

$$
\left\{\begin{array}{l}
i \partial_{t} u-\Delta u=0 \\
\left.u\right|_{t=0}=f .
\end{array}\right.
$$

The solution $u$ to (1.8) is given by $u(\mathbf{x}, t):=e^{-i t \Delta} f(\mathbf{x})$. Then, the rescaled function $u^{\lambda}(\mathbf{x}, t):=u\left(\lambda \mathbf{x}, \lambda^{2} t\right), \lambda>0$, is also a solution to (1.8) but with the rescaled initial condition $f^{\lambda}(\mathbf{x}):=f(\lambda \mathbf{x})$. Noting that $\operatorname{supp} \widehat{f^{\lambda}}=\lambda \cdot \operatorname{supp} \widehat{f}$, it is easy to see that the power of $N$ in (1.5) is the only power that is consistent with this scaling. We point out that the inequalities (1.3), (1.6), and (1.7) are also scaling-invariant with respect to this scaling associated to the linear Schrödinger equation and that the scaling-invariance shows sharpness of these estimates.

When $M$ is a compact manifold, the Strichartz estimate (1.2) becomes much more difficult and much less is known. This is partially due to the fact that we do not have the dispersive estimate (1.4) on a compact manifold. Moreover, (1.2) requires deep understanding of the eigenvalues and the eigenfunctions of the Laplacian. In the following, we focus on the case when $M$ is a standard flat torus $\mathbb{T}^{d}=(\mathbb{R} / \mathbb{Z})^{d}$, corresponding to the usual periodic boundary condition. Moreover, we restrict our attention to the diagonal case, i.e. $q=r$. Then, one would like to establish the following scaling-invariant 2 Strichartz estimate:

$$
\left\|e^{-i t \Delta} f\right\|_{L_{t, \mathbf{x}}^{p}\left(I \times \mathbb{T}^{d}\right)} \lesssim N^{\frac{d}{2}-\frac{d+2}{p}}\|f\|_{L^{2}\left(\mathbb{T}^{d}\right)},
$$

for all $f \in L^{2}\left(\mathbb{T}^{d}\right)$ with supp $\widehat{f} \subset[-N, N]^{d}$, where $I$ is a compact interval. Note that, in the compact setting, an estimate of the form (1.9) does not hold with $I=\mathbb{R}$, unless $p=\infty$.

By drawing an analogy to the Euclidean case $M=\mathbb{R}^{d}$, one may hope to have (1.9) for $p \geq \frac{2(d+2)}{d}$. By combining the tools from number theory, such as a divisor counting argument and the Hardy-Littlewood circle method, and the Tomas-Stein restriction method from harmonic analysis, Bourgain [3] proved (1.9) for certain ranges of $p$ : (i) $p>\frac{2(d+2)}{d}$ when $d=1,2$, (ii) $p>4$ when $d=3$, and (iii) $p>\frac{2(d+4)}{d}$ for higher dimensions $d \geq 4$. It is

\footnotetext{
${ }^{2}$ Obviously, the scaling associated to the linear Schrödinger equation discussed above for $\mathbb{R}^{d}$ does not quite make sense on $\mathbb{T}^{d}$. We nonetheless call the estimate (1.9) scaling-invariant.
} 
worthwhile to note that, when $d=1,2$, (1.9) is known to fail at the endpoint $p=\frac{2(d+2)}{d}$. See [3, 37]. Namely, the situation on $\mathbb{T}^{d}$ is strictly worse than the Euclidean setting. Indeed, Bourgain [3, 6] conjectured that

$$
\left\|e^{-i t \Delta} f\right\|_{L_{t, \mathbf{x}}^{p}\left(I \times \mathbb{T}^{d}\right)} \leq K_{p, N}\|f\|_{L^{2}\left(\mathbb{T}^{d}\right)}
$$

for all $f \in L^{2}\left(\mathbb{T}^{d}\right)$ with supp $\widehat{f} \subset[-N, N]^{d}$, where $K_{p, N}$ satisfies

$$
\begin{cases}K_{p, N}<c_{p}, & \text { if } p<\frac{2(d+2)}{d}, \\ K_{p, N} \ll N^{\varepsilon}, & \text { if } p=\frac{2(d+2)}{d}, \\ K_{p, N}<c_{p} N^{\frac{d}{2}-\frac{d+2}{p}}, & \text { if } p>\frac{2(d+2)}{d},\end{cases}
$$

for any small $\varepsilon>0$. Here, $A(N) \ll B(N)$ means that $\lim _{N \rightarrow \infty} \frac{A(N)}{B(N)}=0$. More recently, using multilinear restriction theory after [1, 8], Bourgain [7] improved the result (iii) for $d \geq 4$ and showed that (1.9) holds for $p>\frac{2(d+3)}{d}$. The general conjecture (1.11), however, remains open up to date. In [3, 4, 21, 42, these Strichartz estimates were then applied to prove well-posedness results of NLS (1.1) on $\mathbb{T}^{d}$. See Subsection 1.3 for more on the well-posedness issue of (1.1).

Let us conclude this subsection by stating the result by Herr [20]. He considered the quintic NLS on a three-dimensional Zoll manifold $M$, i.e. a compact Riemannian manifold such that all geodesics are simple and closed with a common minimal period. One simplest example is the three dimensional sphere $\mathbb{S}^{3}$. By establishing the Strichartz estimate (1.9) on $M$ (instead of $\mathbb{T}^{d}$ ) with $p>4$, he proved local well-posedness of the quintic NLS on a three-dimensional Zoll manifold $M$ in the energy space $H^{1}(M)$. As mentioned above, all geodesics on a Zoll manifold have a common minimal period. Hence, it is natural to ask if a Strichartz estimate of the form (1.9) holds on a manifold, where there is no common minimal period for geodesics. This leads us to the study of Strichartz estimates on an irrational torus $\mathbb{T}_{\boldsymbol{\alpha}}^{d}$, since it is one of the simplest examples of manifolds with no common minimal period for geodesics.

1.2. Strichartz estimate on irrational tori. In the remaining part of this paper, we focus on the case when $M$ is an irrational torus $\mathbb{T}_{\boldsymbol{\alpha}}^{d}$ :

$$
M=\mathbb{T}_{\boldsymbol{\alpha}}^{d}:=\prod_{j=1}^{d} \mathbb{R} /\left(\alpha_{j} \mathbb{Z}\right), \quad \alpha_{j}>0, j=1, \ldots, d .
$$

As the name suggests, we are mainly interested in the case when at least one $\alpha_{j}$ is irrational. More generally, we are interested in the case when at least one $\alpha_{j}$ is "rationally independent" of the remaining ones, i.e. there exists $\alpha_{j}$ that can not be written as a linear combination of the other $\alpha_{k}$ 's with rational coefficients.

First consider the case when all $\alpha_{j}$ 's are rational. Namely, $M=\mathbb{T}_{\boldsymbol{\alpha}}^{d}$ is a "rational" torus. In this case, the problem can be reduced to that on the standard torus $\mathbb{T}^{d}$ by a simple geometric consideration. By writing $\alpha_{j}=\frac{k_{j}}{m_{j}}$ for some $k_{j}, m_{j} \in \mathbb{N}$, let $k$ be the least common multiple of $k_{j}$ 's. The basic idea is to view the scaled standard torus 
$\widetilde{M}:=k \mathbb{T}^{d}=(\mathbb{R} /(k \mathbb{Z}))^{d}$ as a disjoint union of parallel translates of the original rational torus $M=\mathbb{T}_{\boldsymbol{\alpha}}^{d}$ with $\alpha_{j}^{-1} k$ copies in the $x_{j}$-direction.

Now, consider the linear Schrödinger equation (1.8) on $M=\mathbb{T}_{\boldsymbol{\alpha}}^{d}$. By periodic extension, we can view this problem on the scaled standard torus $\widetilde{M}=k \mathbb{T}^{d}$. Given an initial condition $f$ and the solution $u(t)=e^{-i t \Delta} f$ on $M$, let $\widetilde{f}$ and $\widetilde{u}$ denote their periodic extensions on $\widetilde{M}$, respectively. By uniqueness of solutions to the linear Schrödinger equation, we see that $\widetilde{u}(t)=e^{-i t \Delta} \widetilde{f}$ on $\widetilde{M}$. Clearly, the Strichartz estimates on the standard torus $\mathbb{T}^{d}$ also hold on the scaled standard torus $\widetilde{M}=k \mathbb{T}^{d}$, where the implicit constants further depend on $k$. With $\boldsymbol{\alpha}=\left(\alpha_{1}, \ldots, \alpha_{d}\right)$, we have

$$
\|\widetilde{f}\|_{L_{\mathbf{x}}^{2}(\widetilde{M})}=C(\boldsymbol{\alpha})\|f\|_{L_{\mathbf{x}}^{2}(M)} \quad \text { and } \quad\|\widetilde{u}(t)\|_{L_{\mathbf{x}}^{p}(\widetilde{M})}=C(\boldsymbol{\alpha}, p)\|u(t)\|_{L_{\mathbf{x}}^{p}(M)} .
$$

Moreover, letting $\widehat{f}$ and $\tilde{\mathcal{F}}[\tilde{f}]$ denote the Fourier coefficients of $f$ on $\mathbb{T}_{\boldsymbol{\alpha}}^{d}$ and $\tilde{f}$ on $k \mathbb{T}^{d}$, respectively, we have

$$
\begin{aligned}
\widehat{f}(\mathbf{n}) & =\frac{1}{\left|\mathbb{T}_{\boldsymbol{\alpha}}^{d}\right|} \int_{\mathbb{T}_{\boldsymbol{\alpha}}^{d}} f(\mathbf{x}) e^{-2 \pi i \sum_{j=1}^{d} n_{j} \frac{x_{j}}{\alpha_{j}}} d \mathbf{x} \\
& =\frac{1}{\left|k \mathbb{T}^{d}\right|} \int_{k \mathbb{T}^{d}} \widetilde{f}(\mathbf{x}) e^{-2 \pi i \sum_{j=1}^{d} \frac{k n_{j}}{\alpha_{j}} \frac{x_{j}}{k}} d \mathbf{x}=\widetilde{\mathcal{F}}[\widetilde{f}]\left(\frac{k}{\alpha_{1}} n_{1}, \ldots, \frac{k}{\alpha_{d}} n_{d}\right),
\end{aligned}
$$

where $\mathbf{n}=\left(n_{1}, \ldots, n_{d}\right) \in \mathbb{Z}^{d}$ and $|\cdot|$ denotes the Lebesgue measure of a set. Namely, we have

$$
\operatorname{supp} \tilde{\mathcal{F}}[\widetilde{f}]=\frac{k}{\alpha} \cdot \operatorname{supp} \widehat{f}:=\left\{\left(\frac{k}{\alpha_{1}} n_{1}, \ldots, \frac{k}{\alpha_{d}} n_{d}\right) \in \mathbb{Z}^{d}: \mathbf{n} \in \operatorname{supp} \widehat{f}\right\} .
$$

Therefore, we see that the Strichartz estimates of the form (1.10) on the standard torus $\mathbb{T}^{d}$ also hold on our rational torus $M=\mathbb{T}_{\boldsymbol{\alpha}}^{d}$, where the implicit constants further depends on $\boldsymbol{\alpha}=\left(\alpha_{1}, \ldots, \alpha_{d}\right)$. When there is no $\alpha_{j}$ that is rationally independent of the remaining ones, we can use spatial and temporal dilations to reduce the situation to the case of a rational torus above. Therefore, in the following, we assume that at least one $\alpha_{j}$ is rationally independent of the remaining $\alpha_{k}$ 's.

Before proceeding further, let us change the spatial domain $M=\mathbb{T}_{\boldsymbol{\alpha}}^{d}$ to the standard torus $\mathbb{T}^{d}$ at the expense of modifying the Laplacian. By a change of spatial variables $\left(x_{j} \mapsto \alpha_{j} x_{j}\right)$, we see that (1.1) is equivalent to the following NLS on the usual torus $\mathbb{T}^{d}=(\mathbb{R} / \mathbb{Z})^{d}$ :

$$
\left\{\begin{array}{l}
i \partial_{t} u-\Delta u= \pm|u|^{p-1} u \\
\left.u\right|_{t=0}=u_{0} \in H^{s}\left(\mathbb{T}^{d}\right),
\end{array} \quad(\mathbf{x}, t) \in \mathbb{T}^{d} \times \mathbb{R},\right.
$$

where the Laplace operator $\Delta$ is now defined by

$$
\widehat{\Delta f}(\mathbf{n})=-4 \pi^{2} Q(\mathbf{n}) \widehat{f}(\mathbf{n})
$$

with $\mathbf{n}=\left(n_{1}, \ldots, n_{d}\right) \in \mathbb{Z}^{d}$ and

$$
Q(\mathbf{n})=\theta_{1} n_{1}^{2}+\cdots+\theta_{d} n_{d}^{2}, \quad \frac{1}{C} \leq \theta_{j}:=\frac{1}{\alpha_{j}^{2}} \leq C, j=1, \cdots, d .
$$

We point out that some estimates in the following depend on $C$ in (1.15) but not on the specific arithmetic nature of $\theta_{j}$ 's.

Our main interest is to discuss well-posedness of the Cauchy problem (1.13) by first studying relevant Strichartz estimates in this setting. As compared to the problem on the 
standard torus $\mathbb{T}^{d}$, i.e. with $Q(\mathbf{n})=|\mathbf{n}|^{2}=\sum_{j=1}^{d} n_{j}^{2}$, it is a lot harder to study Strichartz estimates on irrational tori. The main reason for this difficulty is that the number theoretic tools such as a divisor counting argument and the Hardy-Littlewood circle method do not work well in this setting.

Previously, Bourgain [6] and Catoire-Wang [11] studied the Cauchy problem (1.13) on irrational tori and proved some local well-posedness results in subcritical Sobolev spaces. See Theorem 1.4 below. In the following, we investigate new Strichartz estimates on irrational tori and use them to prove well-posedness results of the Cauchy problem (1.13) in both subcritical and critical Sobolev spaces. In the rest of the paper, we assume that the Laplacian $\Delta$ is defined by (1.14), unless stated otherwise, and define the linear Schrödinger evolution by 3

$$
e^{-i t \Delta} f(\mathbf{x})=\sum_{\mathbf{n} \in \mathbb{Z}^{d}} \widehat{f}(\mathbf{n}) e^{2 \pi i(\mathbf{n} \cdot \mathbf{x}+Q(\mathbf{n}) t)},
$$

where $Q(\mathbf{n})$ is as in (1.15). We first summarize the known Strichartz estimates. In the following, $I$ denotes a compact interval in $\mathbb{R}$.

Theorem 1.1. The Strichartz estimate on a irrational torus is known to hold

$$
\left\|e^{-i t \Delta} f\right\|_{L_{t, \mathbf{x}}^{p}\left(I \times \mathbb{T}^{d}\right)} \lesssim K_{p, N}\|f\|_{L^{2}\left(\mathbb{T}^{d}\right)},
$$

for all $f \in L^{2}\left(\mathbb{T}^{d}\right)$ with supp $\widehat{f} \subset[-N, N]^{d}$ in the following cases:

(i) $d=2[11]^{4}: K_{4, N}=N^{\frac{1}{6}}$,

(ii) $d=3[6]: K_{4, N}=N^{\frac{1}{3}+\varepsilon}$,

(iii) $d \geq 3$ [11]: $K_{4, N}=N^{\frac{d}{4}-\frac{d}{2(d+1)}+\varepsilon}$ when $d$ is odd, and $K_{4, N}=N^{\frac{d}{4}-\frac{1}{2}+\varepsilon}$ when $d$ is even,

(iv) $d \geq 2\left[7: K_{p, N}=N^{\varepsilon}\right.$ for $p=\frac{2(d+1)}{d}$,

for any small $\varepsilon>0$.

Note that the implicit constants in (1.17) depend on $C$ in (1.15) and the length of the local-in-time interval $I$. The same comment applies to all the estimates in the remaining of the paper and we do not mention this dependence explicitly in the following. In [6], Bourgain also proved

$$
\left\|e^{-i t \Delta} f\right\|_{L_{t}^{p} L_{\mathbf{x}}^{4}\left(I \times \mathbb{T}^{3}\right)} \lesssim N^{\frac{3}{4}-\frac{2}{p}}\|f\|_{L^{2}\left(\mathbb{T}^{3}\right)}
$$

for $p>\frac{16}{3}$.

In this paper, we partially improve the known results in Theorem 1.1, and obtain some critical Strichartz estimates when $p$ is large. We state our main result on the Strichartz estimates on irrational tori.

\footnotetext{
${ }^{3}$ Strictly speaking, there is an extra factor of $2 \pi$ in front of $Q(\mathbf{n})$ in (1.15). However, such a factor can be eliminated by time dilation and thus, for simplicity of notations, we drop it in the following.

4 After the completion of this manuscript, we learned that this result in 11] was recently improved to $K_{4, N}=N^{\frac{131}{832}+}$ by Demirbas [15]. While the proof in [11] is based on Jarník's argument [27, the proof in [15] is based on Huxley's counting estimate 24]. More recently, this result in 115 was improved to $K_{4, N}=N^{\frac{1}{8}+}$ by Demeter [14. See the footnote in Theorem 1.2 (ii).
} 
Theorem 1.2. (i) The following scaling-invariant Strichartz estimate holds on an irrational torus:

$$
\left\|e^{-i t \Delta} f\right\|_{L_{t, \mathbf{x}}^{p}\left(I \times \mathbb{T}^{d}\right)} \lesssim N^{\frac{d}{2}-\frac{d+2}{p}}\|f\|_{L^{2}\left(\mathbb{T}^{d}\right)}
$$

for all $f \in L^{2}\left(\mathbb{T}^{d}\right)$ with supp $\widehat{f} \subset[-N, N]^{d}$, provided that $d$ and $p$ are in the following ranges:

(i.a) $d=2: p>\frac{20}{3}$,

(i.b) $d=3: p>\frac{16}{3}$,

(i.c) $d=4: p>4$,

(i.d) $d \geq 5: p \geq 4$.

(ii) Let $\varepsilon>0$. Then, the Strichartz estimate with an $\varepsilon$-loss of regularity holds on an irrational torus 5

$$
\left\|e^{-i t \Delta} f\right\|_{L_{t, \mathbf{x}}^{p}\left(I \times \mathbb{T}^{d}\right)} \lesssim N^{\frac{d}{2}-\frac{d+2}{p}+\varepsilon}\|f\|_{L^{2}\left(\mathbb{T}^{d}\right)}
$$

for all $f \in L^{2}\left(\mathbb{T}^{d}\right)$ with supp $\widehat{f} \subset[-N, N]^{d}$, provided that $d$ and $p$ are in the following ranges:

(ii.a) $d=2: p \geq \frac{20}{3}$,

(ii.b) $d=3: p=\frac{16}{3}$,

(ii.c) $d=4: p=4$.

When $d \geq 3$, we follow a relatively simple argument after Bourgain [6] and prove Theorem 1.2 in Subsection 2.1. When $d=2$, this argument proves (1.20) only for $p \geq 8$. In Subsection 2.2, we present a duality argument to prove (1.19) (when $d=2$ ) for $p>12$. In Section 3, we establish certain level set estimates and provide a full proof of Theorem 1.2 when $d=2$.

By interpolating with Theorems 1.1 and 1.2, we can obtain Strichartz estimates for other values of $p$. The following corollary shows a summary of known Strichartz estimates on irrational tori at this point.

Corollary 1.3. Let $\varepsilon>0$. Then, the following Strichartz estimates hold for all $f \in L^{2}\left(\mathbb{T}^{d}\right)$ with $\operatorname{supp} \widehat{f} \subset[-N, N]^{d}$ :

\footnotetext{
${ }^{5}$ In a very recent preprint, Demeter 14 proved the Strichartz estimates (1.20) with an $\varepsilon$-loss on the standard torus $\mathbb{T}^{d}$ for $p \geq \frac{2(d+3)}{d}$. His argument is based on incidence geometry, without any number theory. As a result, the same result holds for irrational tori and hence improves our result in Theorem 1.2 (ii) in a significant manner. This also improves the values of $s_{0}$ in some subcritical local well-posedness results below (Theorem 1.4(i.a), (i.b), (ii.a), and (ii.b)). Note that the result in 14] comes with an $\varepsilon$-loss and thus it does not improve the scaling-invariant Strichartz estimate (1.19) in Theorem 1.2 (i) and critical local well-posedness results in Theorems 1.5 and 1.6
} 
(i) $d=2$ :

$$
\left\|e^{-i t \Delta} f\right\|_{L_{t, \mathbf{x}}^{p}\left(I \times \mathbb{T}^{2}\right)} \lesssim \begin{cases}N^{\varepsilon}\|f\|_{L^{2}\left(\mathbb{T}^{2}\right)}, & \text { for } 2<p \leq 3, \\ N^{\frac{2}{3}-\frac{2}{p}+\varepsilon}\|f\|_{L^{2}\left(\mathbb{T}^{2}\right)}, & \text { for } 3<p<4, \\ N^{\frac{1}{6}}\|f\|_{L^{2}\left(\mathbb{T}^{2}\right)}, & \text { for } p=4, \\ N^{\frac{3}{4}-\frac{7}{3 p}+\varepsilon}\|f\|_{L^{2}\left(\mathbb{T}^{2}\right)}, & \text { for } 4<p \leq \frac{20}{3}, \\ N^{1-\frac{4}{p}}\|f\|_{L^{2}\left(\mathbb{T}^{2}\right)}, & \text { for } p>\frac{20}{3} .\end{cases}
$$

(ii) $d=3$ :

$$
\left\|e^{-i t \Delta} f\right\|_{L_{t, \mathbf{x}}^{p}\left(I \times \mathbb{T}^{3}\right)} \lesssim \begin{cases}N^{\varepsilon}\|f\|_{L^{2}\left(\mathbb{T}^{3}\right)}, & \text { for } 2<p \leq \frac{8}{3}, \\ N^{1-\frac{8}{3 p}+\varepsilon}\|f\|_{L^{2}\left(\mathbb{T}^{3}\right)}, & \text { for } \frac{8}{3}<p \leq 4, \\ N^{\frac{5}{4}-\frac{11}{3 p}+\varepsilon}\|f\|_{L^{2}\left(\mathbb{T}^{3}\right)}, & \text { for } 4<p \leq \frac{16}{3} \\ N^{\frac{3}{2}-\frac{5}{p}}\|f\|_{L^{2}\left(\mathbb{T}^{3}\right)}, & \text { for } p>\frac{16}{3}\end{cases}
$$

(iii) $d=4$ :

$$
\left\|e^{-i t \Delta} f\right\|_{L_{t, \mathbf{x}}^{p}\left(I \times \mathbb{T}^{4}\right)} \lesssim \begin{cases}N^{\varepsilon}\|f\|_{L^{2}\left(\mathbb{T}^{4}\right)}, & \text { for } 2<p \leq \frac{5}{2}, \\ N^{\frac{4}{3}-\frac{10}{3 p}+\varepsilon}\|f\|_{L^{2}\left(\mathbb{T}^{4}\right)}, & \text { for } \frac{5}{2}<p \leq 4, \\ N^{2-\frac{6}{p}}\|f\|_{L^{2}\left(\mathbb{T}^{4}\right)}, & \text { for } p>4\end{cases}
$$

(iv) $d \geq 5$ :

$$
\left\|e^{-i t \Delta} f\right\|_{L_{t, \mathbf{x}}^{p}\left(I \times \mathbb{T}^{d}\right)} \lesssim \begin{cases}N^{\varepsilon}\|f\|_{L^{2}\left(\mathbb{T}^{d}\right)}, & \text { for } 2<p \leq \frac{2(d+1)}{d}, \\ N^{\left(\frac{d}{4}-\frac{1}{2}\right)\left(\frac{2 d}{d-1}-\frac{4(d+1)}{p(d-1)}\right)+\varepsilon}\|f\|_{L^{2}\left(\mathbb{T}^{d}\right)}, & \text { for } \frac{2(d+1)}{d}<p<4, \\ N^{\frac{d}{2}-\frac{d+2}{p}}\|f\|_{L^{2}\left(\mathbb{T}^{d}\right)}, & \text { for } p \geq 4 .\end{cases}
$$

1.3. Local well-posedness results of NLS on irrational tori. In the following, we apply these Strichartz estimates in Corollary 1.3 to the Cauchy problem of NLS on an irrational torus:

$$
\left\{\begin{array}{l}
i \partial_{t} u-\Delta u= \pm|u|^{2 k} u \\
\left.u\right|_{t=0}=u_{0} \in H^{s}\left(\mathbb{T}^{d}\right),
\end{array} \quad(\mathbf{x}, t) \in \mathbb{T}^{d} \times \mathbb{R},\right.
$$

where $k \in \mathbb{N}$ is a positive integer and the Laplacian $\Delta$ is defined by (1.14). First, recall the following notion. When $M=\mathbb{R}^{d}$, the Cauchy problem (1.1) enjoys the dilation symmetry. Namely, if $u$ is a solution to (1.1) with respect to an initial condition $u_{0}$, then the rescaled function $u_{\lambda}(\mathbf{x}, t):=\lambda^{\frac{2}{p-1}} u\left(\lambda \mathbf{x}, \lambda^{2} t\right)$ is also a solution to (1.1) with the rescaled initial condition $u_{0, \lambda}(\mathbf{x}):=\lambda^{\frac{2}{p-1}} u_{0}(\lambda \mathbf{x})$. We say that the Sobolev index $s_{c}$ is critical if the homogeneous Sobolev norm $\|\cdot\|_{\dot{H}^{s_{c}\left(\mathbb{R}^{d}\right)}}$ is invariant under this dilation symmetry. In particular, the critical Sobolev index is given by $s_{c}=\frac{d}{2}-\frac{2}{p-1}$. When $M \neq \mathbb{R}^{d}$, we may not have this natural dilation symmetry. Nonetheless, the notion of the critical Sobolev index provides us important heuristics. In terms of the Cauchy problem (1.25), the critical 
Sobolev index $s_{c}$ is given by

$$
s_{c}=\frac{d}{2}-\frac{1}{k}
$$

First, we state local well-posedness in subcritical Sobolev spaces $H^{s}\left(\mathbb{T}^{d}\right)$ with $s>s_{c}$.

Theorem 1.4 (Local well-posedness in subcritical spaces). Let $d \geq 2$ and $k \in \mathbb{N}$. Then, there exists $s_{0}=s_{0}(k, d)$ such that the Cauchy problem (1.25) on a d-dimensional irrational torus $\mathbb{T}^{d}$ is locally well-posed in $H^{s}\left(\mathbb{T}^{d}\right)$ for $s>s_{0}$ in the following cases:

(i) $d=2$ :

(i.a) $k=1, s_{0}=\frac{1}{3}$ [11],

(i.b) $k=2,3,4,5, s_{0}=\frac{7 k-3}{7 k+5}$,

(i.c) $k \geq 5, s_{0}=s_{c}=1-\frac{1}{k}$,

Note that the values of $s_{0}$ in (i.b) and (i.c) coincide when $k=5$.

(ii) $d=3$ :

(ii.a) $k=1, s_{0}=\frac{2}{3}[6]$,

(ii.b) $k=2, s_{0}=\frac{53}{52}$,

(ii.c) $k \geq 3, s_{0}=s_{c}=\frac{3}{2}-\frac{1}{k}$,

(iii) $d \geq 4: k \geq 1, s_{0}=s_{c}=\frac{d}{2}-\frac{1}{k}$.

After Bourgain's seminal paper [3], the Fourier restriction norm method, involving the $X^{s, b}$-space, has been applied to study well-posedness of a wide class of equations. In our proof, we also employ the $X^{s, b}$-spaces and by the standard argument, the proof is reduced to establishing certain multilinear Strichartz estimates.

Furthermore, by applying the well-posedness theory involving the $U^{p}$ - and $V^{p}$-spaces developed by Tataru, Koch, and their collaborators [30, 18, 21, 22, we prove some critical local well-posedness 6

Theorem 1.5 (Local well-posedness in critical spaces). Given $d \geq 2$ and $k \in \mathbb{N}$, let $s_{c}$ be the critical Sobolev index given by (1.26). Then, the Cauchy problem (1.25) on a ddimensional irrational torus $\mathbb{T}^{d}$ is locally well-posed in the critical Sobolev space $H^{s_{c}}\left(\mathbb{T}^{d}\right)$ in the following cases:

(i) $d=2: k \geq 6$,

(ii) $d=3: k \geq 3$,

(iii) $d \geq 4: k \geq 2$.

Once again, the proof is reduced to establishing certain multilinear Strichartz estimates. See Propositions 5.6 and 5.7 .

Lastly, we briefly discuss the case of a partially irrational torus. Namely, we consider Strichartz estimates on an irrational torus $\mathbb{T}_{\boldsymbol{\alpha}}^{d}$, when some of $\alpha_{j}$ 's in (1.12) are rationally

\footnotetext{
${ }^{6}$ In a very recent paper, Strunk 35] extended Theorem 1.5 to (i) $k \geq 3$ when $d=2$ and (ii) $k=2$ when $d=3$. The main idea in 35] is based on considering Strichartz estimates in mixed Lebesgue spaces $L_{t}^{q} L_{\mathbf{x}}^{r}$ to improve the multilinear Strichartz estimate (Proposition 5.7 below). This clever argument avoids the need of improving the scaling-invariant Strichartz estimate (1.19).
} 
dependent. In this case, we may obtain improvements over Theorem 1.2, yielding better local well-posedness results than those presented in Theorems 1.4 and 1.5. For simplicity of presentation, we only consider an example of the three-dimensional torus of the form $\mathbb{T}^{2} \times \mathbb{T}_{\alpha_{3}}$, where two periods are the same. By a change of spatial variables as before, we consider the Cauchy problem (1.25), where the multiplier $Q(\mathbf{n})$ in (1.15) is given by

$$
Q(\mathbf{n})=n_{1}^{2}+n_{2}^{2}+\theta_{3} n_{3}^{2}, \quad \theta_{3}>0
$$

i.e. we set $\theta_{1}=\theta_{2}=1$. Then, we have the following local well-posedness result for the energy-critical quintic NLS on a three-dimensional partially irrational torus.

Theorem 1.6. Suppose that $Q(\mathbf{n})$ is given by (1.27). Then, the energy-critical quintic $N L S$, (1.25) with $k=2$, on $\mathbb{T}^{3}$ is locally well-posed in the critical Sobolev space $H^{1}\left(\mathbb{T}^{3}\right)$.

Previously, Herr-Tataru-Tzvetkov [21] proved local well-posedness in the energy space $H^{1}\left(\mathbb{T}^{3}\right)$ of the energy-critical quintic NLS on the three-dimensional standard torus $\mathbb{T}^{3}$. By combining the results in [7] and [22], we also see that the energy-critical cubic NLS on the four-dimensional standard torus $\mathbb{T}^{4}$ is local well-posedness in the energy space $H^{1}\left(\mathbb{T}^{4}\right)$. See also the work by the third author [42] for some other critical local well-posedness results. The result in [42], however, does not cover an energy-critical setting. As mentioned earlier, Herr 20] proved local well-posedness in the energy space of the energy-critical quintic NLS on three-dimensional Zoll manifolds. We point out that Theorem 1.6 seems to be the first local well-posedness result of the energy-critical NLS in its energy space $H^{1}\left(\mathbb{T}^{3}\right)$, where there is no common minimal period for geodesics.

We present a sketch of the proof in Appendix B. More precisely, we revisit the argument in Section 3 and prove the sharp Strichartz estimate (1.19) on $\mathbb{T}^{3}$ for $p>\frac{14}{3}$ under the assumption (1.27). The rest follows from a slight modification of the argument in Section 5. Lastly, note that Theorem 1.6 combined with the conservation of mass and Hamiltonian yields small data global well-posedness of the quintic NLS, (1.25) with $k=2$, in $H^{1}\left(\mathbb{T}^{3}\right)$, just as in [21, 20]. Recently, global well-posedness (for large data) of the energy-critical quintic NLS on the three-dimensional standard torus $\mathbb{T}^{3}$ and on the three-dimensional sphere $\mathbb{S}^{3}$ was obtained by Ionescu-Pausader [26] and Pausader-Tzvetkov-Wang [32, respectively. It would be of interest to investigate if global well-posedness of the energy-critical quintic NLS holds in the setting of Theorem $[1.6$

This paper is organized as follows. In Section 2, we prove Theorem 1.2 when $d \geq 3$ and partially when $d=2$, via multilinear estimates and a duality argument. In Section 3, we establish certain level set estimates and prove Theorem 1.2 when $d=2$. In Section 4, we prove local well-posedness results in subcritical Sobolev spaces (Theorem 1.4). In Section 5 , we prove local well-posedness results in critical Sobolev spaces (Theorem 1.5). In Appendix $\mathrm{A}$, we present a proof of (2.1) below, using the Hardy-Littlewood circle method. In Appendix B, we sketch a proof of Theorem 1.6.

\footnotetext{
${ }^{7}$ After Strunk's result 35, it is now of interest to study global well-posedness of the energy-critical quintic NLS on a general three-dimensional irrational torus in its energy space $H^{1}\left(\mathbb{T}^{3}\right)$.
} 
Acknowledgments: Z. Guo is supported in part by NNSF of China (No.11371037, No.11271023) and Beijing Higher Education Young Elite Teacher Project. Y. Wang is supported by NNSF of China (No.11126247, No.11201143) and AARMS Postdoctoral Fellowship. The authors would like to thank the anonymous referee for thoughtful comments that have significantly improved the introduction of this paper. T. Oh would like to express his sincere gratitude to Professor Harold N. Shapiro for his support and teaching in mathematics, including the Hardy-Littlewood circle method used in this paper, as well as in life.

\section{Strichartz estimates: Part 1}

In this section, we prove our main result (Theorem 1.2) for $d \geq 3$ and present a partial proof for $d=2$. In [6], Bourgain treated the three-dimensional case. His argument is based on the following estimate:

$$
\int_{\mathbb{T}}\left|\sum_{0 \leq n \leq N} e^{2 \pi i n^{2} t}\right|^{r} d t \sim N^{r-2}
$$

for $r>4$. We first apply this argument and generalize the result in [6] to a general dimension $d \geq 3$. When $r=4$, (2.1) holds with a logarithmic loss (Hua's inequality). This yields the endpoint case for $d=3,4$. When $d=2$, this also proves Theorem 1.2 (ii) but only for $p \geq 8$. In Subsection 2.2 , we present a simple duality argument when $d=2$. This proves Theorem 1.2 (i) for $p>12$. The full proof of Theorem 1.2 for $d=2$, i.e. (1.19) for $p>\frac{20}{3}$ and (1.20) for $p \geq \frac{20}{3}$, is presented in Section 3.

2.1. Higher dimensional case: $d \geq 3$. In this subsection, we prove Theorem 1.2 when $d \geq 3$. First, we prove the following lemma, which can be viewed as a version of HausdorffYoung's inequality.

Lemma 2.1 (Hausdorff-Young's inequality). Let $d \geq 2$ and $\mathbf{a} \in \mathbb{Z}^{d}$. Given a sequence $\left\{c_{\mathbf{n}}\right\}_{\mathbf{n} \in \mathbb{Z}^{d}}$, define $F_{\mathbf{a}}(t)$ by

$$
F_{\mathbf{a}}(t)=\sum_{\mathbf{n} \in \mathbb{Z}^{d}} c_{\mathbf{n}} c_{\mathbf{a}-\mathbf{n}} e^{2 \pi i[Q(\mathbf{n})+Q(\mathbf{a}-\mathbf{n})] t},
$$

where $Q(\mathbf{n})$ is as in (1.15). Then, for $p \geq 2$, we have

$$
\left\|F_{\mathbf{a}}(t)\right\|_{L_{t}^{p}([-1,1])} \lesssim\left[\sum_{k \in \mathbb{Z}}\left(\sum_{|Q(\mathbf{n})+Q(\mathbf{a}-\mathbf{n})-k| \leq \frac{1}{2}}\left|c_{\mathbf{n}} c_{\mathbf{a}-\mathbf{n}}\right|\right)^{p^{\prime}}\right]^{\frac{1}{p^{\prime}}}
$$

where $\frac{1}{p}+\frac{1}{p^{\prime}}=1$.

Lemma 2.1 was used in [6] for the three-dimensional case. See also Lemma 2 in [11. A proof for general dimensions in [11] relies on Schur's lemma. In the following, we give a direct proof for reader's convenience. 
Proof. When $p=\infty$, (2.3) follows immediately. Hence, by interpolation, it suffices to prove (2.3) for $p=2$. Let $\eta(t)$ be a cutoff function supported on $[-2,2]$ such that $\eta \equiv 1$ on $[-1,1]$. By Plancherel identity, we have

$$
\begin{aligned}
\left\|F_{\mathbf{a}}(t)\right\|_{L_{t}^{2}([-1,1])} & \leq\left\|F_{\mathbf{a}}(t) \eta(t)\right\|_{L_{t}^{2}(\mathbb{R})} \\
& =\left\|\sum_{\mathbf{n}} c_{\mathbf{n}} c_{\mathbf{a}-\mathbf{n}} \widehat{\eta}(\tau-[Q(\mathbf{n})+Q(\mathbf{a}-\mathbf{n})])\right\|_{L_{\tau}^{2}} \\
& =\left\|\sum_{k \in \mathbb{Z}} \sum_{n \in I_{k, \mathbf{a}}} c_{\mathbf{n}} c_{\mathbf{a}-\mathbf{n}} \widehat{\eta}(\tau-[Q(\mathbf{n})+Q(\mathbf{a}-\mathbf{n})])\right\|_{L_{\tau}^{2}} \\
& =\left\|\sum_{k \in \mathbb{Z}} B_{k}(\tau)\right\|_{L_{\tau}^{2}},
\end{aligned}
$$

where $I_{k, \mathbf{a}}=\left\{\mathbf{n} \in \mathbb{Z}^{d}: Q(\mathbf{n})+Q(\mathbf{a}-\mathbf{n})-k \in\left(-\frac{1}{2}, \frac{1}{2}\right]\right\}$ and

$$
B_{k}(\tau)=\sum_{\mathbf{n} \in I_{k, \mathbf{a}}} c_{\mathbf{n}} c_{\mathbf{a}-\mathbf{n}} \widehat{\eta}(\tau-[Q(\mathbf{n})+Q(\mathbf{a}-\mathbf{n})])
$$

Noting that $\left|B_{k}(\tau)\right| \lesssim \sum_{n \in I_{k, \mathbf{a}}}\left|c_{\mathbf{n}} c_{\mathbf{a}-\mathbf{n}}\right|\langle\tau-k\rangle^{-2}$, we have

$$
\begin{aligned}
& \left\|\sum_{k \in \mathbb{Z}} B_{k}(\tau)\right\|_{L_{\tau}^{2}}^{2}=\left\|\left(\sum_{k \in \mathbb{Z}} B_{k}(\tau)\right)^{2}\right\|_{L_{\tau}^{1}} \lesssim \sum_{k, k^{\prime}}\left\|B_{k}(\tau) B_{k}^{\prime}(\tau)\right\|_{L_{\tau}^{1}} \\
& \quad \lesssim \sum_{k, k^{\prime}} \sum_{\mathbf{n} \in I_{k, \mathbf{a}}} \sum_{\mathbf{n}^{\prime} \in I_{k^{\prime}, \mathbf{a}}}\left|c_{\mathbf{n}} c_{\mathbf{a}-\mathbf{n}}\right|\left|c_{\mathbf{n}^{\prime}} c_{\mathbf{a}-\mathbf{n}^{\prime}}\right| \int_{\mathbb{R}}\langle\tau-k\rangle^{-2}\left\langle\tau-k^{\prime}\right\rangle^{-2} d \tau \\
& \quad \lesssim \sum_{k, k^{\prime}} \frac{1}{\left\langle k-k^{\prime}\right\rangle^{2}} \sum_{\mathbf{n} \in I_{k, \mathbf{a}}} \sum_{\mathbf{n}^{\prime} \in I_{k^{\prime}, \mathbf{a}}}\left|c_{\mathbf{n}} c_{\mathbf{a}-\mathbf{n}}\right|\left|c_{\mathbf{n}^{\prime}} c_{\mathbf{a}-\mathbf{n}^{\prime}}\right|
\end{aligned}
$$

By Cauchy-Schwarz inequality (in $k$ ) followed by Young inequality, we have

$$
\begin{aligned}
& \leq\left[\sum_{k}\left(\sum_{\mathbf{n} \in I_{k, \mathbf{a}}}\left|c_{\mathbf{n}} c_{\mathbf{a}-\mathbf{n}}\right|\right)^{2}\right]^{\frac{1}{2}}\left[\sum_{k}\left(\sum_{k^{\prime}} \sum_{\mathbf{n}^{\prime} \in I_{k^{\prime}, \mathbf{a}}} \frac{\left|c_{\mathbf{n}^{\prime}} c_{\mathbf{a}-\mathbf{n}^{\prime}}\right|}{\left\langle k-k^{\prime}\right\rangle^{2}}\right)^{2}\right]^{\frac{1}{2}} \\
& \lesssim \sum_{k}\left(\sum_{\mathbf{n} \in I_{k, \mathbf{a}}}\left|c_{\mathbf{n}} c_{\mathbf{a}-\mathbf{n} \mid}\right|\right)^{2} .
\end{aligned}
$$

This completes the proof of Lemma 2.1.

Next, we state the main proposition. Theorem 1.2 then follows this proposition and Bernstein's inequality when $d \geq 3$.

Proposition 2.2. Let $f$ be a function on $\mathbb{T}^{d}$ with $\operatorname{supp} \widehat{f} \subset[-N, N]^{d}$.

(i) Let $d \geq 3$. Then, for $p \geq \max \left(\frac{16}{d}+, 4\right)$, we have

$$
\left\|e^{-i t \Delta} f\right\|_{L_{t, \mathrm{loc}}^{p} L_{\mathbf{x}}^{4}} \lesssim N^{\frac{d}{4}-\frac{2}{p}}\|f\|_{L^{2}}
$$


(ii) Suppose that $d$ and $p$ satisfy (ii.a) $d=2, p \geq 8$, (ii.b) $(d, p)=\left(3, \frac{16}{3}\right)$, or (ii.c) $(d, p)=(4,4)$. Then, we have

$$
\left\|e^{-i t \Delta} f\right\|_{L_{t, \mathrm{loc}}^{p} L_{\mathbf{x}}^{4}} \lesssim N^{\frac{d}{4}-\frac{2}{p}}(\log N)^{\frac{2}{q}}\|f\|_{L^{2}},
$$

where $q=p$ when $d=3,4$ and $q=8$ when $d=2$.

Bourgain proved (2.4) for $d=3$. See Proposition 1.1 in [6]. Our proof follows the ideas developed in [6]. By setting $p=4$ when $d \geq 5$ and $p=\frac{16}{d}+$ when $d=3$, 4, Proposition 2.2 yields the $L^{4}$-Strichartz estimate, which improves the result in [11] for $d \geq 3$. Note that the Strichartz estimate (2.4) is essentially sharp in higher dimensions $(d \geq 4)$. Indeed, on $\mathbb{R}^{d}$ with $d \geq 2$, by Sobolev inequality and interpolation of (1.6) and (1.7)

$$
\left\|e^{-i t \Delta} f\right\|_{L_{t}^{p} L_{x}^{4}\left(\mathbb{R} \times \mathbb{R}^{d}\right)} \lesssim N^{2\left(\frac{1}{4}-\frac{1}{p}\right)}\left\|e^{-i t \Delta} f\right\|_{L_{t, x}^{4}\left(\mathbb{R} \times \mathbb{R}^{d}\right)} \lesssim N^{\frac{d}{4}-\frac{2}{p}}\|f\|_{L^{2}\left(\mathbb{R}^{d}\right)},
$$

for $p \geq 4$.

We first use Proposition 2.2 to prove Theorem 1.2 when $d \geq 3$.

Proof of Theorem 1.2 for $d \geq 3$. Suppose that $p \geq \max \left(\frac{16}{d}+, 4\right)$, satisfying the hypothesis of Theorem 1.2 (i). By Bernstein's inequality and Proposition 2.2 (i), we have

$$
\left\|e^{-i t \Delta} f\right\|_{L_{t, \mathbf{x}}^{p}} \lesssim N^{\frac{d}{4}-\frac{d}{p}}\left\|e^{-i t \Delta} f\right\|_{L_{t}^{p} L_{\mathbf{x}}^{4}} \lesssim N^{\frac{d}{2}-\frac{d+2}{p}}\|f\|_{L^{2}} .
$$

By repeating the same argument with Proposition 2.2 (ii), we obtain Theorem 1.2 (ii) when $d=3,4$. When $d=2$, this yields Theorem 1.2 (ii) only for $p \geq 8$.

We now present the proof of Proposition 2.2 .

Proof of Proposition 2.2. (i) Let $Q(\mathbf{n})$ be as in (1.15). Then, we have

$$
\left(e^{-i t \Delta} f\right)(\mathbf{x})=\sum_{\mathbf{n} \in \mathbb{Z}^{d}} \widehat{f}(\mathbf{n}) e^{2 \pi i(\mathbf{n} \cdot \mathbf{x}+Q(\mathbf{n}) t)}
$$

With $c_{\mathbf{n}}=\widehat{f}(\mathbf{n})$, let $F_{\mathbf{a}}(t)$ be as in (2.2). Then, by Minkowski's integral inequality with $p \geq 4$, we have

$$
\begin{aligned}
\left\|e^{-i t \Delta} f\right\|_{L_{t}^{p} L_{\mathbf{x}}^{4}}^{2} & =\left\|\left(e^{-i t \Delta} f\right)^{2}\right\|_{L_{t}^{\frac{p}{2}} L_{\mathbf{x}}^{2}}=\left\|\left(\sum_{\mathbf{a} \in \mathbb{Z}^{d}}\left|F_{\mathbf{a}}(t)\right|^{2}\right)^{\frac{1}{2}}\right\|_{L_{t}^{\frac{p}{2}}} \\
& \leq\left(\sum_{\mathbf{a} \in \mathbb{Z}^{d}}\left\|F_{\mathbf{a}}(t)\right\|_{L_{t}^{\frac{p}{2}}}^{2}\right)^{1 / 2} .
\end{aligned}
$$

For $\ell \in \mathbb{Z}$, let $A_{\ell}=\left\{\mathbf{n} \in \mathbb{Z}^{d}:|Q(\mathbf{n})-\ell| \leq 1\right\} \cap[-N, N]^{d}$. Noting that $Q(\mathbf{n})+Q(\mathbf{a}-\mathbf{n})=$ $\frac{1}{2}(Q(2 \mathbf{n}-\mathbf{a})+Q(\mathbf{a}))$, the condition $|Q(\mathbf{n})+Q(\mathbf{a}-\mathbf{n})-k| \leq \frac{1}{2}$ is equivalent to $2 \mathbf{n} \in \mathbf{a}+A_{\ell}$ with $\ell=2 k-Q(\mathbf{a})$. Note that, $\left|\left\{\ell \in \mathbb{Z}: 2 \mathbf{n} \in \mathbf{a}+A_{\ell}\right\}\right| \lesssim 1$ for all $\mathbf{n} \in \mathbb{Z}^{d}$. Then, by 
Lemma 2.1 and Cauchy-Schwarz and Hölder's inequalities, we have

$$
\begin{aligned}
\left\|F_{\mathbf{a}}\right\|_{L_{t}^{\frac{p}{2}}} & \leq\left[\sum_{\ell \in \mathbb{Z}}\left(\sum_{2 \mathbf{n} \in \mathbf{a}+A_{\ell}}\left|c_{\mathbf{n}} c_{\mathbf{a}-\mathbf{n}}\right|\right)^{\frac{p}{p-2}}\right]^{\frac{p-2}{p}} \\
& \lesssim\left[\sum_{\ell}\left|A_{\ell}\right|^{\frac{p}{2(p-2)}}\left(\sum_{2 \mathbf{n} \in \mathbf{a}+A_{\ell}} \mid c_{\mathbf{n}} c_{\mathbf{a}-\left.\mathbf{n}\right|^{2}}\right)^{\frac{p}{2(p-2)}}\right]^{\frac{p-2}{p}} \\
& \leq\left(\sum_{\ell}\left|A_{\ell}\right|^{\frac{p}{p-4}}\right)^{\frac{p-4}{2 p}}\left(\sum_{\ell} \sum_{2 \mathbf{n} \in \mathbf{a}+A_{\ell}} \mid c_{\mathbf{n}} c_{\mathbf{a}-\left.\mathbf{n}\right|^{2}}\right)^{\frac{1}{2}} \\
& \sim\left(\sum_{\ell}\left|A_{\ell}\right|^{\frac{p}{p-4}}\right)^{\frac{p-4}{2 p}}\left(\sum_{\mathbf{n} \in \mathbb{Z}^{d}}\left|c_{\mathbf{n}} c_{\mathbf{a}-\mathbf{n}}\right|^{2}\right)^{\frac{1}{2}} .
\end{aligned}
$$

From (2.7) and (2.8), we have

$$
\left\|e^{-i t \Delta} f\right\|_{L_{t}^{p} L_{\mathbf{x}}^{4}} \leq C\left(\sum_{\ell}\left|A_{\ell}\right|^{\frac{p}{p-4}}\right)^{\frac{p-4}{4 p}}\|f\|_{L^{2}} .
$$

Now, let $\eta(t)$ be a smooth function with a compact support $I \subset \mathbb{R}$ such that $\widehat{\eta} \geq 0$ and $\widehat{\eta} \geq 1$ on $[-1,1]$. Now we estimate $\left(\sum_{\ell}\left|A_{\ell}\right|^{\frac{p}{p-4}}\right)^{\frac{p-4}{4 p}}$, using

$$
\left|A_{\ell}\right| \leq \int\left[\sum_{\substack{\mathbf{n} \in \mathbb{Z}^{d} \\\left|n_{j}\right| \leq N}} e^{2 \pi i Q(\mathbf{n}) t}\right] \eta(t) e^{-2 \pi i \ell t} d t
$$

If $p \leq 8$, then we have $\frac{p}{p-4} \geq 2$. Then, by Hausdorff-Young's inequality, we have

$$
\begin{aligned}
& \left(\sum_{\ell}\left|A_{\ell}\right|^{\frac{p}{p-4}}\right)^{\frac{p-4}{4 p}} \lesssim\left[\int_{I} \prod_{j=1}^{d}\left|\sum_{\left|n_{j}\right| \leq N} e^{2 \pi i \theta_{j} n_{j}^{2} t}\right|^{\frac{p}{4}} d t\right]^{\frac{1}{p}} \\
& \lesssim \prod_{j=1}^{d}\left[\int_{I} \mid \sum_{\left|n_{j}\right| \leq N} e^{\left.2 \pi i \theta_{j} n_{j}^{2} t\right|^{\frac{d p}{4}}} d t\right]^{\frac{1}{d p}} \lesssim\left[\int_{I}\left|\sum_{0 \leq n \leq N} e^{2 \pi i n^{2} t}\right|^{\frac{d p}{4}} d t\right]^{\frac{1}{p}} .
\end{aligned}
$$

Note that $r=\frac{d p}{4}>4$, since $p>\frac{16}{d}$. Then, by an application of the Hardy-Littlewood circle method (see Appendix $\mathrm{A}$ ), we have

$$
\int_{I}\left|\sum_{0 \leq n \leq N} e^{2 \pi i n^{2} t}\right|^{r} d t \sim N^{r-2}
$$

yielding (2.10) $\lesssim N^{\frac{d}{4}-\frac{2}{p}}$. Hence, (2.4) follows from (2.9) in this case.

If $p>8$, then by Bernstein's inequality (in $t$ ), we have

$$
\left\|e^{-i t \Delta} f\right\|_{L_{t}^{p} L_{\mathbf{x}}^{4}} \leq C N^{\frac{1}{4}-\frac{2}{p}}\left\|e^{-i t \Delta} f\right\|_{L_{t}^{8} L_{\mathbf{x}}^{4}} .
$$

Then, (2.4) follows from (2.12) and (2.4) for $p=8$. 
(ii) When $(d, p)=(2,8),\left(3, \frac{16}{3}\right)$, or $(4,4)$, we have $r=\frac{d p}{4}=4$. In this case, (2.11) does not hold. Nonetheless, by Hua's inequality [40], we have

$$
\int_{I}\left|\sum_{0 \leq n \leq N} e^{2 \pi i n^{2} t}\right|^{4} d t \lesssim N^{2}(\log N)^{2}
$$

See also [25, (8.13)]. Then, (2.5) follows from (2.13) and repeating the computation in (i). This completes the proof of Proposition 2.2 .

2.2. Two dimensional case. For $d=2$, the sharp estimate (1.19) is not covered by Proposition 2.2. In the following, we use a simple duality argument and prove the sharp Strichartz estimate (1.19) for $p>12$. Without loss of generality, we assume that

$$
Q(\mathbf{n})=n_{1}^{2}+\theta n_{2}^{2}, \quad \frac{1}{C} \leq \theta \leq C .
$$

Then, the local-in-time Strichartz estimate can be expressed as

$$
\left\|\sum_{\mathbf{n} \in S_{N}} a_{\mathbf{n}} e^{2 \pi i(\mathbf{n} \cdot \mathbf{x}+Q(\mathbf{n}) t)}\right\|_{L_{t, \mathbf{x}}^{p}\left(I \times \mathbb{T}^{2}\right)} \leq K_{p, N}\left(\sum_{\mathbf{n} \in S_{N}}\left|a_{\mathbf{n}}\right|^{2}\right)^{1 / 2},
$$

where $S_{N}$ denotes the following set:

$$
S_{N}:=\left\{\left(n_{1}, n_{2}\right) \in \mathbb{Z}^{2}:\left|n_{j}\right| \leq N, j=1,2\right\} .
$$

Our task is to seek for an optimal constant $K_{p, N}$. By duality, (2.15) is equivalent to

$$
\left(\sum_{\mathbf{n} \in S_{N}}|\widehat{f}(\mathbf{n}, Q(\mathbf{n}))|^{2}\right)^{\frac{1}{2}} \leq K_{p, N}\|f\|_{L_{t, \mathbf{x}}^{p^{\prime}}\left(I \times \mathbb{T}^{2}\right)},
$$

for any $f \in L^{p^{\prime}}\left(I \times \mathbb{T}^{2}\right)$, where $\frac{1}{p}+\frac{1}{p^{\prime}}=1$. Here, the Fourier transform $\widehat{f}$ is defined by

$$
\widehat{f}(\mathbf{n}, \tau)=\int_{\mathbb{R}} \int_{\mathbb{T}^{2}} e^{-2 \pi i \mathbf{n} \cdot \mathbf{x}} e^{-2 \pi i \tau t} \mathbf{1}_{I}(t) f(\mathbf{x}, t) d \mathbf{x} d t .
$$

Then, (1.19) for $p>12$ follows once we prove the next proposition.

Proposition 2.3. For $p>12$, we have $K_{p, N} \lesssim N^{1-\frac{4}{p}}$. Namely, we have

$$
\left(\sum_{\mathbf{n} \in S_{N}}|\widehat{f}(\mathbf{n}, Q(\mathbf{n}))|^{2}\right)^{\frac{1}{2}} \lesssim N^{1-\frac{4}{p}}\|f\|_{L_{t, \mathbf{x}}^{p^{\prime}}\left(I \times \mathbb{T}^{2}\right)}^{2} .
$$

Remark 2.4. Recall that, on the standard torus $\mathbb{T}^{2}$, i.e. with $Q(\mathbf{n})=n_{1}^{2}+n_{2}^{2}$, Bourgain [3] proved $K_{p, N} \lesssim N^{1-\frac{4}{p}}$ for $p>4$. Hence, Proposition 2.3 states that, on an irrational torus, the same estimate for $K_{p, N}$ holds, but only for $p>12$.

Proof. Without loss of generality, assume that $I$ is centered at 0 . Let $\mathbf{R}$ be a kernel defined by

$$
\mathbf{R}(\mathbf{x}, t)=\sum_{\mathbf{n} \in S_{N}} e^{2 \pi i(\mathbf{n} \cdot \mathbf{x}+Q(\mathbf{n}) t)}
$$

Then, defining $R_{\theta}$ by

$$
R_{\theta}(x, t)=\sum_{|n| \leq N} e^{2 \pi i\left(n x+\theta n^{2} t\right)}
$$


we have $\mathbf{R}(\mathbf{x}, t)=R_{1}\left(x_{1}, t\right) R_{\theta}\left(x_{2}, t\right)$. From Proposition 3.114 in [3], we have

$$
\left\|R_{1}(x, t)\right\|_{L_{t, x}^{p}(I \times \mathbb{T})} \leq C_{p, I} N^{1-\frac{3}{p}}
$$

for $p>6$. Bourgain's argument is based on an application of the Hardy-Littlewood circle method. See also Lemma 2.4 in 23 . for a simpler proof based on the Poisson summation formula. Note that (2.21) does not hold for $p=6$. See Rogovskaya [33] and [3].

By Hölder's inequality, (2.21), and Sobolev inequality, we have

$$
\begin{aligned}
\|\mathbf{R}\|_{L_{t, \mathbf{x}}^{p}\left(I \times \mathbb{T}^{2}\right)} & =\left(\int_{I}\left\|R_{1}\left(x_{1}, t\right)\right\|_{L_{x_{1}}^{p}}^{p}\left\|R_{\theta}\left(x_{2}, t\right)\right\|_{L_{x_{2}}^{p}}^{p} d t\right)^{\frac{1}{p}} \\
& \leq\left\|R_{1}\left(x_{1}, t\right)\right\|_{L_{t, x_{1}}^{p}}\left\|R_{\theta}\left(x_{2}, t\right)\right\|_{L_{t}^{\infty}\left(I ; L_{x_{2}}^{p}\right)} \\
& \lesssim N^{1-\frac{3}{p}}\left\|R_{\theta}\left(x_{2}, t\right)\right\|_{L_{t}^{\infty}\left(I ; H_{x_{2}}^{\frac{1}{2}-\frac{1}{p}}\right)} \lesssim N^{2-\frac{4}{p}} .
\end{aligned}
$$

By (2.19), Young's inequality, and (2.22), we have

$$
\begin{aligned}
\sum_{\mathbf{n} \in S_{N}}|\widehat{f}(\mathbf{n}, Q(\mathbf{n}))|^{2} & =\left\langle\mathbf{R} * \mathbf{1}_{I} f, \mathbf{1}_{I} f\right\rangle \leq\|\mathbf{R}\|_{L_{t, \mathbf{x}}^{\frac{p}{2}}\left(2 I \times \mathbb{T}^{2}\right)}\|f\|_{L_{t, \mathbf{x}}^{p^{\prime}}\left(I \times \mathbb{T}^{2}\right)}^{2} \\
& \lesssim N^{2-\frac{8}{p}}\|f\|_{L_{t, \mathbf{x}}^{p^{\prime}}\left(I \times \mathbb{T}^{2}\right)}^{2}
\end{aligned}
$$

as long as $p>12$.

\section{Strichartz estimates: Part 2}

3.1. Level set estimates. In this section, we prove Theorem 1.2 when $d=2$. The main ingredient is the level set estimates on irrational tori in Proposition 3.1 below. For level sets estimates on the usual torus $\mathbb{T}^{d}$, see [3, 23]. It turns out that these level set estimates are useful only when $d=2,3$ (see Remark 3.3), but we state and prove the results for a general dimension. In the following, we assume that $\theta_{1}=1$ in (1.15) for simplicity. Namely, we consider

$$
Q(\mathbf{n})=n_{1}^{2}+\theta_{2} n_{2}^{2}+\cdots+\theta_{d} n_{d}^{2}
$$

Also, let $S_{N}=\left\{\mathbf{n} \in \mathbb{Z}^{d}:\left|n_{j}\right| \leq N, j=1, \ldots, d\right\}$.

Proposition 3.1. Let I be a compact interval in $\mathbb{R}$. Given

$$
f(\mathbf{x})=\sum_{\mathbf{n} \in S_{N}} c_{\mathbf{n}} e^{2 \pi i \mathbf{n} \cdot \mathbf{x}}
$$

such that $\left\|c_{\mathbf{n}}\right\|_{\ell_{\mathbf{n}}^{2}}=1$, define the distribution function $A_{\lambda}$ by

$$
A_{\lambda}=\left\{(\mathbf{x}, t) \in \mathbb{T}^{2} \times I:\left|\left(e^{-i t \Delta} f\right)(\mathbf{x}, t)\right|>\lambda\right\} .
$$

(i) For any $\varepsilon>0$, we have

$$
\left|A_{\lambda}\right| \lesssim N^{2(d-1) \frac{1}{1+6 \varepsilon}} \lambda^{-6+\frac{24}{1+6 \varepsilon} \varepsilon}
$$

for $\lambda \gtrsim N^{\frac{d}{2}-\frac{1}{4}+\varepsilon}$.

(ii) Let $q>6$. Then, there exists small $\varepsilon>0$ such that

$$
\left|A_{\lambda}\right| \lesssim N^{\frac{d}{2} q-(d+2)} \lambda^{-q}
$$


for $\lambda \gtrsim N^{\frac{d}{2}-\varepsilon}$.

In (3.4) and (3.5), the implicit constants depend on $\varepsilon>0, q>6$, and $|I|$, but are independent of $f$.

We present the proof of Proposition 3.1 in Subsections 3.2 and 3.3. In the following, we use Proposition 3.1 to prove Theorem 1.2 when $d=2$. First, we present the proof of Theorem 1.2 (ii.a), i.e. we prove (1.20) for $p \geq \frac{20}{3}$ when $d=2$. Recall that Catoire-Wang [11] proved

$$
\left\|e^{-i t \Delta} f\right\|_{L_{t, \mathbf{x}}^{4}\left(I \times \mathbb{T}^{d}\right)} \lesssim N^{\frac{1}{6}}\|f\|_{L^{2}\left(\mathbb{T}^{d}\right)}
$$

for $f \in \mathbb{T}^{2}$ with supp $\widehat{f} \subset[-N, N]^{2}$. Given $f$ as in (3.2), let $F(\mathbf{x}, t)=e^{-i t \Delta} f(\mathbf{x}, t)$. By Cauchy-Schwarz inequality, we have $\|F\|_{L_{t, \mathbf{x}}^{\infty}} \lesssim N$. Then, with Proposition 3.1(i) and (3.6), we have

$$
\begin{aligned}
\int_{I \times \mathbb{T}^{2}}|F(\mathbf{x}, t)|^{p} d \mathbf{x} d t & \leq \int_{N^{\frac{3}{4}+\varepsilon} \lesssim|F| \lesssim N}|F(\mathbf{x}, t)|^{p} d \mathbf{x} d t+N^{\left(\frac{3}{4}+\varepsilon\right)(p-4)} \int|F(\mathbf{x}, t)|^{4} d \mathbf{x} d t \\
& \lesssim N^{2-\frac{12}{1+6 \varepsilon} \varepsilon} \int_{N^{\frac{3}{4}+}}^{N} \lambda^{p-7+\frac{24}{1+6 \varepsilon} \varepsilon} d \lambda+N^{\left(\frac{3}{4}+\varepsilon\right)(p-4)+\frac{2}{3}} \\
& \lesssim N^{p-4+},
\end{aligned}
$$

where the last inequality holds as long as $p \geq \frac{20}{3}$. This proves Theorem 1.2 (ii.a). By Proposition 3.1 (i) and (ii), Theorem 1.2 (i.a) follows in a similar manner. We omit details.

Remark 3.2. When $d=2$, Proposition 3.1 (i) and (ii) basically says that the level set estimates (3.4) and (3.5) are sufficient in proving the Strichartz estimates (1.19) and (1.20) for $p>6$ as long as $\lambda$ is large: $\lambda \geq N^{\frac{1}{4}+}$. Hence, an improvement on Theorem 1.2 when $d=2$ may be obtained if we can improve the lower bound on $\lambda$ in Proposition 3.1 (i) or the $L^{4}$-Strichartz estimate (3.6).

Remark 3.3. In [7, Bourgain proved

$$
\left\|e^{-i t \Delta} f\right\|_{L_{t, \mathbf{x}}^{p}\left(I \times \mathbb{T}^{d}\right)} \lesssim N^{\varepsilon}\|f\|_{L^{2}\left(\mathbb{T}^{d}\right)}
$$

for $p=\frac{2(d+1)}{d}$. See Proposition 8 and the comment afterward in [7]. Combining Proposition 3.1 (i) and (3.7), we obtain (1.20) only for $p \geq \frac{2(3 d+1)}{d}$. When $d=2$, the combination of Proposition 3.1 (i) and (3.6) yields a better result. When $d \geq 3$, Proposition 2.2 yields better results. We point out that, when $d=3$, combining Proposition 3.1(i) with Theorem 1.1 (ii) yields another proof of Theorem 1.2 (ii.b).

3.2. Proof of Proposition 3.1 (i). Let $\eta$ be a smooth cutoff function supported on $\left[\frac{1}{200}, \frac{1}{100}\right]$. Given $q \in \mathbb{N}$, define $J_{q}$ by

$$
J_{q}=\{a \in \mathbb{N}: 1 \leq a \leq q,(a, q)=1\} .
$$

Then, for given $M \in \mathbb{N}$ with $M \geq N$, we define

$$
\Phi(t)=\sum_{M \leq q<2 M} \sum_{a \in J_{q}} \eta\left(q^{2}\left\|t-\frac{a}{q}\right\|\right),
$$


where $\|x\|=\min _{n \in \mathbb{Z}}|x-n|$ denotes the distance of $x$ to the closest integer. Note that $\Phi$ is periodic with period 1. By taking a Fourier transform, we have

$$
\widehat{\Phi}(k)=\sum_{M \leq q<2 M} \frac{1}{q^{2}} c_{q}(k) \widehat{\eta}\left(q^{-2} k\right)
$$

where $c_{q}(k)$ denotes Ramanujan's sum: $c_{q}(k):=\sum_{a \in J_{q}} e^{-2 \pi i \frac{a}{q} k}$. Let $\phi(q)$ be the Euler's totient function defined by $\phi(q)=\sum_{a \in J_{q}} 1$. Then, by Theorem 330 in [19], we have

$$
\widehat{\Phi}(0) \sim \frac{1}{M^{2}} \sum_{M \leq q<2 M} \phi(q) \sim 1
$$

Namely, $\widehat{\Phi}(0)$ is independent of $M$.

Without loss of generality, assume that $I$ is centered at 0 . With $Q(\mathbf{n})$ in (3.1), define $\mathbf{R}$ as in (2.19), where $S_{N}=\left\{\mathbf{n} \in \mathbb{Z}^{d}:\left|n_{j}\right| \leq N, j=1, \ldots, d\right\}$. Then, we have $\mathbf{R}(\mathbf{x}, t)=$ $R_{1}\left(x_{1}, t\right) \prod_{j=2}^{d} R_{\theta_{j}}\left(x_{j}, t\right)$, where $R_{\theta}$ is defined in (2.20). Now, letting $\chi$ be a smooth cutoff function support on $3 I$ such that $\chi(t) \equiv 1$ on $2 I$, define $\mathbf{R}_{1}$ and $\mathbf{R}_{2}$ by

$$
\mathbf{R}_{1}(\mathbf{x}, t)=\frac{\Phi(t)}{\widehat{\Phi}(0)} \mathbf{R}(\mathbf{x}, t) \chi(t) \quad \text { and } \quad \mathbf{R}_{2}(\mathbf{x}, t)=\mathbf{R}(\mathbf{x}, t) \chi(t)-\mathbf{R}_{1}(\mathbf{x}, t)
$$

Noting that the intervals $I_{\ell, q, a}:=\left[\ell+\frac{a}{q}+\frac{1}{200 q^{2}}, \ell+\frac{a}{q}+\frac{1}{100 q^{2}}\right]$ are disjoint for distinct values of $\ell, a$, and $q \sim M \gg 1$, it follows from Weyl's inequality [31, Theorem 1 on p. 41] that

$$
\left|R_{1}\left(x_{1}, t\right)\right| \lesssim \frac{N}{q^{\frac{1}{2}}}+N^{\frac{1}{2}}(\log q)^{\frac{1}{2}}+q^{\frac{1}{2}}(\log q)^{\frac{1}{2}} \lesssim M^{\frac{1}{2}}(\log M)^{\frac{1}{2}}
$$

for $t \in I_{\ell, q, a}$ since $q \sim M \geq N$. Then, along with a trivial bound $\left|R_{\theta_{j}}\left(x_{j}, t\right)\right| \lesssim N$, we obtain

$$
\left\|\mathbf{R}_{1}\right\|_{L_{t, \mathbf{x}}^{\infty}} \lesssim \min \left(N^{d-1} M^{\frac{1}{2}}(\log M)^{\frac{1}{2}}, N^{d}\right)
$$

Next, we consider $\mathbf{R}_{2}$. By expanding $\Phi(t)$ in the Fourier series, we have

$$
\mathbf{R}_{2}(\mathbf{x}, t)=-\frac{1}{\widehat{\Phi}(0)} \mathbf{R}(\mathbf{x}, t) \chi(t) \sum_{k \neq 0} \widehat{\Phi}(k) e^{2 \pi i k t}
$$

First, recall the following lemma (Lemma 3.33 in [3]). Given $M \in \mathbb{N}$ and $k \in \mathbb{Z}$, we have

$$
\sum_{M \leq q<2 M}\left|c_{q}(k)\right| \lesssim d(k, M) M^{1+}
$$


where $d(k, M)$ denotes the number of divisors of $k$ less than $M$. Then, by taking a Fourier transform of (3.13) with (3.9), (3.14), and $d(k, M) \lesssim k^{0+}$, we have

$$
\begin{aligned}
\left|\widehat{\mathbf{R}}_{2}(\mathbf{n}, \tau)\right| & =\left|\mathbf{1}_{S_{N}}(\mathbf{n}) \sum_{k \neq 0} \frac{\widehat{\Phi}(k)}{\widehat{\Phi}(0)} \widehat{\chi}(\tau-Q(\mathbf{n})-k)\right| \\
& \lesssim \frac{1}{M^{2}} \sum_{k \neq 0} \sum_{M \leq q<2 M}\left|c_{q}(k)\right|\left|\widehat{\eta}\left(q^{-2} k\right) \widehat{\chi}(\tau-Q(\mathbf{n})-k)\right| \\
& \lesssim \frac{1}{M^{2}} \sum_{k \neq 0} k^{0+} M^{1+}\left(\frac{M^{2}}{k}\right)^{0+} \frac{1}{\langle\tau-Q(\mathbf{n})-k\rangle^{10}} \\
& \lesssim M^{-1+} .
\end{aligned}
$$

Define $\Theta_{\lambda}(\mathbf{x}, t)$ by

$$
\Theta_{\lambda}(\mathbf{x}, t)=\exp \left(i \arg \left(e^{-i t \Delta} f(\mathbf{x})\right)\right) \cdot \mathbf{1}_{A_{\lambda}}(\mathbf{x}, t) .
$$

Note that $\operatorname{supp} \Theta_{\lambda}(\mathbf{x}, \cdot) \subset I$ for each $\mathbf{x} \in \mathbb{T}^{d}$. Then, by Cauchy-Schwarz inequality with (3.2), we have

$$
\begin{aligned}
\lambda^{2}\left|A_{\lambda}\right|^{2} & \leq\left(\int_{I \times \mathbb{T}^{2}}\left(e^{-i t \Delta} f\right)(\mathbf{x}, t) \overline{\Theta_{\lambda}(\mathbf{x}, t)} d \mathbf{x} d t\right)^{2}=\left(\sum_{\mathbf{n} \in S_{N}} c_{\mathbf{n}} \overline{\widehat{\Theta}_{\lambda}(\mathbf{n}, Q(\mathbf{n}))}\right)^{2} \\
& \leq \sum_{\mathbf{n} \in S_{N}}\left|\widehat{\Theta}_{\lambda}(\mathbf{n}, Q(\mathbf{n}))\right|^{2}=\left\langle\mathbf{R} * \Theta_{\lambda}, \Theta_{\lambda}\right\rangle=\left\langle(\mathbf{R} \chi) * \Theta_{\lambda}, \Theta_{\lambda}\right\rangle
\end{aligned}
$$

By (3.10), (3.12), and (3.15), we have

$$
\begin{aligned}
& =\left\langle\mathbf{R}_{1} * \Theta_{\lambda}, \Theta_{\lambda}\right\rangle+\left\langle\mathbf{R}_{2} * \Theta_{\lambda}, \Theta_{\lambda}\right\rangle \\
& \leq\left\|\mathbf{R}_{1}\right\|_{L_{t, \mathbf{x}}^{\infty}}\left\|\Theta_{\lambda}\right\|_{L_{t, \mathbf{x}}^{1}}^{2}+\left\|\widehat{\mathbf{R}}_{2}\right\|_{L_{\tau}^{\infty} \ell_{\mathbf{n}}^{\infty}}\left\|\Theta_{\lambda}\right\|_{L_{t, \mathbf{x}}^{2}}^{2} \\
& \leq C_{1} N^{d-1} M^{\frac{1}{2}+\varepsilon_{1}}\left|A_{\lambda}\right|^{2}+M^{-1+\varepsilon_{2}}\left|A_{\lambda}\right|
\end{aligned}
$$

for small $\varepsilon_{1}, \varepsilon_{2}>0$.

Now, choose $M \geq N$ such that

$$
N^{d-1} M^{\frac{1}{2}+\varepsilon_{1}} \sim \lambda^{2} .
$$

The condition (3.19) with $M \geq N$ implies that $\lambda \gtrsim N^{\frac{d}{2}-\frac{1}{4}+\frac{\varepsilon_{1}}{2}}$. Then, (3.18) yields

$$
\left|A_{\lambda}\right| \lesssim\left(\frac{N^{2(d-1)}}{\lambda^{4}}\right)^{\frac{1-\varepsilon_{2}}{1+2 \varepsilon_{1}}} \lambda^{-2} \lesssim N^{2(d-1) \frac{1}{1+3 \varepsilon_{1}}} \lambda^{-6+\frac{12}{1+3 \varepsilon_{1}} \varepsilon_{1}}
$$

by setting $\varepsilon_{2}=\varepsilon_{2}\left(\varepsilon_{1}\right)$ such that $\frac{1-\varepsilon_{2}}{1+2 \varepsilon_{1}}=\frac{1}{1+3 \varepsilon_{1}}$. This proves (3.4) with $\varepsilon=\frac{\varepsilon_{1}}{2}$.

3.3. Proof of Proposition 3.1 (ii). In this subsection, we prove the level set estimate (3.5), which is sharp for $q>6$. The following argument is inspired by Bourgain's paper [3]. We first go over some basic setups, restricting our attention to $t \in \mathbb{T}$.

Let $\left\{\sigma_{n}\right\}_{n \in \mathbb{Z}}$ be the multiplier defined by $\sigma_{n}=1$ on $[-N, N], \sigma_{n}=\frac{N-j}{N}$ for $n=N+j$ and $n=-N-j, j=1, \ldots, N$, and $\sigma_{n}=0$ for $|n| \geq 2 N$. Consider

$$
K(x, t):=\sum_{n \in \mathbb{Z}} \sigma_{n} e^{2 \pi i\left(n x+n^{2} t\right)} .
$$


Then, we have the following lemma. Here, $\|x\|=\min _{n \in \mathbb{Z}}|x-n|$ denotes the distance of $x$ to the closest integer as before.

Lemma 3.4 (Lemma 3.18 in [3]). Let $1 \leq a \leq q \leq N$ and $(a, q)=1$ such that

$$
\left\|t-\frac{a}{q}\right\| \leq \frac{1}{q N}
$$

Then, we have

$$
|K(x, t)| \lesssim \frac{N}{q^{\frac{1}{2}}\left(1+N\left\|t-\frac{a}{q}\right\|^{\frac{1}{2}}\right)} .
$$

Note that the multiplier $\sigma_{n}$ in (3.20) avoids the logarithmic loss (when $q \sim N$ ) in Weyl's inequality (3.11) on the Weyl sum $W_{N}(x, t)=\sum_{|n| \leq N} e^{2 \pi i\left(n x+n^{2} t\right)}$. Indeed, by writing $K=\frac{1}{N} \sum_{k=N}^{2 N-1} W_{k}$, we see that this regularizing effect in (3.22) is analogous to that of the Féjér kernel over the Dirichlet kernel.

In the following, we fix $N \gg 1$, dyadic. For dyadic $M \leq N$, let $\mathcal{R}_{M}$ by

$$
\mathcal{R}_{M}=\left\{\frac{a}{q}: a \in J_{q}, M \leq q<2 M\right\}
$$

where $J_{q}$ is as in (3.8). Let $\psi(t)$ be a smooth cutoff function supported on $\frac{1}{2}+\frac{1}{10} \leq$ $|t| \leq 2-\frac{1}{10}$ such that $\sum_{j \in \mathbb{Z}} \psi\left(2^{-j} t\right)=1$ for $t \neq 0$. For $s \in \mathbb{N}$ with $M \leq 2^{s}<N$ let $\omega_{N, 2^{s}}(t)=\psi\left(2^{s} N t\right)$ and define $\omega_{N, N}$ by

$$
\omega_{N, N}(t)= \begin{cases}\sum_{j \geq \log _{2} N} \psi\left(2^{j} N t\right), & t \neq 0, \\ 1, & t=0 .\end{cases}
$$

Note that we have $\operatorname{supp}\left(\omega_{N, 2^{s}}\right) \subset\left\{|t| \lesssim \frac{1}{2^{s} N}\right\}$ and

$$
\left|\widehat{\omega}_{N, 2^{s}}(k)\right| \lesssim \frac{1}{2^{s} N}\left\langle\frac{k}{2^{s} N}\right\rangle^{-100} .
$$

Now, let

$$
\Omega_{M, N}=\sum_{M \leq 2^{s} \leq N} \omega_{N, 2^{s}}
$$

Then, it follows that $\Omega_{M, N} \equiv 1$ on $\left[-\frac{1}{M N}, \frac{1}{M N}\right]$ and $\operatorname{supp} \Omega_{M, N} \subset\left[-\frac{2}{M N}, \frac{2}{M N}\right]$.

Let $N_{1}=\frac{1}{100} N$. Note that, for $M_{1}<M_{2} \leq N_{1}$, we have

$$
\left(\mathcal{R}_{M_{1}}+\left[-\frac{2}{M_{1} N}, \frac{2}{M_{1} N}\right]\right) \cap\left(\mathcal{R}_{M_{2}}+\left[-\frac{2}{M_{2} N}, \frac{2}{M_{2} N}\right]\right)=\emptyset .
$$

Recall that by Dirichlet's theorem [40, Lemma 2.1], (3.21) is satisfied for all $t \in \mathbb{T}=[0,1]$. Then, by letting $\delta_{T}$ denote the Dirac delta measure at $T$, we have

$$
1=\sum_{\substack{M \leq N_{1} \\ M, \text { dyadic }}} \sum_{T \in \mathcal{R}_{M}} \delta_{T} * \Omega_{M, N}+\rho,
$$

such that $\rho(t) \neq 0$ for some $t \in \mathbb{T}$ implies that $t$ satisfies (3.21) for some $q>N_{1}$. In particular, by Lemma 3.4, we have

$$
|\rho(t) K(x, t)| \lesssim N^{\frac{1}{2}} .
$$


From (3.25) with (3.14), (3.23), and (3.24), we have

$$
\begin{aligned}
|\widehat{\rho}(k)| & \leq\left|\sum_{\substack{M \leq N_{1} \\
M, \text { dyadic }}} \sum_{M \leq 2^{s} \leq N} \mathcal{F}\left[\sum_{T \in \mathcal{R}_{M}} \delta_{T}\right](k) \cdot \widehat{\omega}_{N, 2^{s}}(k)\right| \\
& \lesssim \sum_{\substack{M \leq N_{1} \\
M, \text { dyadic }}} \sum_{M \leq 2^{s} \leq N} \frac{d(k, M) M^{1+}}{2^{s} N}\left\langle\frac{k}{2^{s} N}\right\rangle^{-100} \lesssim N^{-1+}
\end{aligned}
$$

for $k \neq 0$. Here, we used the fact that $d(k, M) \lesssim k^{0+}$ and $M \leq 2^{s} \leq N$.

Now, for each $M$ and $s$, we choose a coefficient $\alpha_{M, s}$ such that

$$
\mathcal{F}\left[\sum_{T \in \mathcal{R}_{M}} \delta_{T} * \omega_{N, 2^{s}}\right](0)=\alpha_{M, s} \widehat{\rho}(0) .
$$

Then, from [3, (3.56)], we have

$$
\left|\alpha_{M, s}\right| \lesssim \frac{M^{2}}{2^{s} N}
$$

Now, we focus on our problem. Namely, we do not assume $t \in \mathbb{T}$ any longer. Given an interval $I \subset \mathbb{R}$, assume that $I$ is centered at 0 and let $\chi$ be a smooth cutoff function support on $3 I$ such that $\chi(t) \equiv 1$ on $2 I$ as before. We define

$$
\begin{aligned}
\mathbf{K}(\mathbf{x}, t) & =\chi(t) \sum_{\mathbf{n} \in \widetilde{S}_{N}} \sigma_{n_{1}} e^{2 \pi i(\mathbf{n} \cdot \mathbf{x}+Q(\mathbf{n}) t)} \\
& =\chi(t) K\left(x_{1}, t\right) \prod_{j=2}^{d} \sum_{\left|n_{j}\right| \leq N} e^{2 \pi i\left(n_{j} x_{j}+\theta_{j} n_{j}^{2} t\right)},
\end{aligned}
$$

where $\widetilde{S}_{N}=\left\{\mathbf{n} \in \mathbb{Z}^{d}:\left|n_{j}\right| \leq N, j=2, \ldots, N\right\}$ and $K\left(x_{1}, t\right)$ is as in (3.20).

Define $\Lambda_{M, s}$ by

$$
\Lambda_{M, s}(\mathbf{x}, t)=\mathbf{K}(\mathbf{x}, t)\left[\sum_{T \in \mathcal{R}_{M}} \delta_{T} * \omega_{N, 2^{s}}(t)-\alpha_{M, s} \rho(t)\right] .
$$

Then, from Lemma 3.4, (3.26), and (3.29) with $M \leq 2^{s} \leq N$, we have

$$
\left|\Lambda_{M, s}(\mathbf{x}, t)\right| \lesssim N^{d-1} \frac{N}{M^{\frac{1}{2}}\left(1+\left(2^{-s} N\right)^{\frac{1}{2}}\right)}+\frac{M^{2}}{2^{s} N} N^{d-\frac{1}{2}} \lesssim N^{d-1}\left(\frac{2^{s} N}{M}\right)^{\frac{1}{2}}
$$

Hence, from (3.32), we have

$$
\left\|f * \Lambda_{M, s}\right\|_{L^{\infty}\left(I \times \mathbb{T}^{d}\right)} \lesssim N^{d-1}\left(\frac{2^{s} N}{M}\right)^{\frac{1}{2}}\|f\|_{L^{1}\left(I \times \mathbb{T}^{d}\right)}
$$

Next, we estimate $\left|\widehat{\Lambda}_{M, s}\right|$. Denote the second factor in (3.31) by $\Phi_{M, s}$, i.e. let

$$
\Phi_{M, s}(t)=\sum_{T \in \mathcal{R}_{M}} \delta_{T} * \omega_{N, 2^{s}}(t)-\alpha_{M, s} \rho(t) .
$$

Note that $\Phi_{M, s}$ is periodic. Moreover, by (3.28), we have $\widehat{\Phi}_{M, s}(0)=0$. Hence, we have

$$
\widehat{\Lambda}_{M, s}(\mathbf{n}, \tau)=\sigma_{n_{1}}\left(\prod_{j=2}^{d} \mathbf{1}_{\left|n_{j}\right| \leq N}\right) \sum_{k \neq 0} \widehat{\Phi}_{M, s}(k) \widehat{\chi}(\tau-Q(\mathbf{n})-k) .
$$


By (3.14), (3.23), (3.27), and (3.29) with $d(k, M) \lesssim k^{0+}$ and $M \leq 2^{s} \leq N$, we have

$$
\left|\widehat{\Phi}_{M, s}(k)\right| \lesssim \frac{d(k, M) M^{1+}}{2^{s} N}\left\langle\frac{k}{2^{s} N}\right\rangle^{-100}+\frac{M^{2}}{2^{s} N^{2-}} \lesssim \frac{M}{2^{s} N^{1-}}
$$

for $k \neq 0$. By summing $|\widehat{\chi}(\tau-Q(\mathbf{n})-k)| \lesssim\langle\tau-Q(\mathbf{n})-k\rangle^{-100}$ over $k \neq 0$, it follows from (3.35) and (3.36) that

$$
\left|\widehat{\Lambda}_{M, s}(\mathbf{n}, \tau)\right| \lesssim \frac{M}{2^{s} N^{1-}}
$$

Hence, from (3.37), we have

$$
\left\|f * \Lambda_{M, s}\right\|_{L^{2}\left(I \times \mathbb{T}^{d}\right)} \lesssim \frac{M}{2^{s} N} N^{0+}\|f\|_{L^{2}\left(I \times \mathbb{T}^{d}\right)}
$$

Also, with the trivial bound $d(k, M) \leq M$ in (3.36), we have

$$
\left\|f * \Lambda_{M, s}\right\|_{L^{2}\left(I \times \mathbb{T}^{d}\right)} \lesssim \frac{M^{2+}}{2^{s} N}\|f\|_{L^{2}\left(I \times \mathbb{T}^{d}\right)} .
$$

The second estimate (3.39) is useful when $M \ll N^{\varepsilon}$.

In the following, we establish another estimate on $\left\|f * \Lambda_{M, s}\right\|_{L^{2}\left(I \times \mathbb{T}^{d}\right)}$, using the following lemma from Bourgain [3].

Lemma 3.5 (Lemma 3.47 in [3]). Let $d(k, M)$ denote the number of divisors of $k$ less than $M$. Then, for any $\beta, B, D>0$, we have

$$
\#\{0 \leq k \leq N: d(k, M)>D\}<c_{\beta, B}\left(D^{-B} M^{\beta} N+M^{B}\right) .
$$

Note that the constant in (3.40) is independent of $D>0, M, N \in \mathbb{N}$.

From (3.35) and (3.36), we have

$$
\begin{gathered}
\left\|f * \Lambda_{M, s}\right\|_{L^{2}\left(I \times \mathbb{T}^{d}\right)} \lesssim \frac{M^{1+}}{2^{s} N}\left(\int \sum_{\widetilde{S}_{N}} \sigma_{n_{1}}^{2}|\widehat{f}(\mathbf{n}, \tau)|^{2}\left[\sum_{k \neq 0} \frac{d(k, M)}{\left\langle\frac{k}{2^{s} N}\right\rangle^{100}\langle\tau-Q(\mathbf{n})-k\rangle^{100}}\right]^{2} d \tau\right)^{\frac{1}{2}} \\
+\frac{M^{2}}{2^{s} N^{2-}}\|f\|_{L^{2}\left(I \times \mathbb{T}^{d}\right)},
\end{gathered}
$$

where $\widetilde{S}_{N}$ is as in (3.30). Given $D>0$ (to be chosen later), separate the first term, depending on $d(k, M) \leq D$ or $>D$. The contribution from $d(k, M) \leq D$ can be estimated by

$$
\lesssim \frac{D M^{1+}}{2^{s} N}\|f\|_{L^{2}\left(I \times \mathbb{T}^{d}\right)}
$$


Next, we estimate the contribution from $d(k, M)>D$. By Cauchy-Schwarz inequality, we have

$$
\begin{aligned}
& {\left[\sum_{k \neq 0} \frac{d(k, M)}{\left\langle\frac{k}{2^{s} N}\right\rangle^{100}\langle\tau-Q(\mathbf{n})-k\rangle^{100}}\right]^{2}} \\
& \quad \leq\left(\sum_{k \neq 0} \frac{d(k, M)^{2}}{\left\langle\frac{k}{2^{s} N}\right\rangle^{200}\langle\tau-Q(\mathbf{n})-k\rangle^{100}}\right)\left(\sum_{\widetilde{k} \neq 0} \frac{1}{\langle\tau-Q(\mathbf{n})-\widetilde{k}\rangle^{100}}\right) \\
& \quad \lesssim\left(\sum_{k \neq 0} \frac{d(k, M)^{2}}{\left\langle\frac{k}{2^{s} N}\right\rangle^{200}\langle\tau-Q(\mathbf{n})-k\rangle^{100}}\right) .
\end{aligned}
$$

Now, we estimate the first term on the right-hand side of (3.41) after applying (3.43). By first integrating in $\tau$, then summing over $\left|n_{j}\right| \lesssim N$ for $j=1, \ldots, d$, applying Lemma 3.5 (with $2 B$ and $2 \beta$ instead of $B$ and $\beta$ ), the trivial bound $d(k, M) \leq M$, and HausdorffYoung's inequality, we have

$$
\begin{aligned}
& \lesssim \frac{M^{1+} N^{\frac{d}{2}}}{2^{s} N}\left(\sum_{|k| \lesssim 2^{s} N} d(k, M)^{2}+\sum_{j=1}^{\infty} \sum_{|k| \sim 2^{s+j} N} d(k, M)^{2}\left\langle\frac{k}{2^{s} N}\right\rangle^{-200}\right)^{\frac{1}{2}}\|\widehat{f}\|_{L_{\tau}^{\infty} \ell_{\mathbf{n}}^{\infty}} \\
& \lesssim \frac{M^{2+} N^{\frac{d}{2}}}{2^{s} N}\left(\sum_{j=0}^{\infty} 2^{-200 j}\left(D^{-2 B} M^{2 \beta} 2^{s+j} N+M^{2 B}\right)\right)^{\frac{1}{2}}\|f\|_{L^{1}\left(I \times \mathbb{T}^{d}\right)} \\
& \lesssim N^{\frac{d}{2}}\left(\frac{D^{-B} M^{2+\beta+}}{2^{\frac{s}{2}} N^{\frac{1}{2}}}+\frac{M^{2+B+}}{2^{s} N}\right)\|f\|_{L^{1}\left(I \times \mathbb{T}^{d}\right)} .
\end{aligned}
$$

Hence, from (3.41), (3.42), and (3.44) with $M \leq N$, we have

$$
\begin{aligned}
\left\|f * \Lambda_{M, s}\right\|_{L^{2}\left(I \times \mathbb{T}^{d}\right)} \lesssim \frac{D M^{1+}}{2^{s} N}\|f\|_{L^{2}\left(I \times \mathbb{T}^{d}\right)} & \\
& +N^{\frac{d}{2}}\left(\frac{D^{-B} M^{2+\beta+}}{2^{\frac{s}{2}} N^{\frac{1}{2}}}+\frac{M^{2+B+}}{2^{s} N}\right)\|f\|_{L^{1}\left(I \times \mathbb{T}^{d}\right)} .
\end{aligned}
$$

Define $\Lambda$ by

$$
\Lambda(\mathbf{x}, t)=\sum_{\substack{M \leq N_{1} \\ M, \text { dyadic }}} \sum_{M \leq 2^{s} \leq N} \Lambda_{M, s}(\mathbf{x}, t),
$$

where $\Lambda_{M, s}$ is as in (3.31). By (3.25), we have

$$
(\mathbf{K}-\Lambda)(\mathbf{x}, t)=\left[1+\sum_{\substack{M \leq N_{1} \\ M, \text { dyadic }}} \sum_{M \leq 2^{s} \leq N} \alpha_{M, s}\right] \mathbf{K}(\mathbf{x}, t) \rho(t) .
$$

Then, by (3.26), (3.29), and (3.30) with $N_{1}=\frac{1}{100} N$, we have

$$
\|\mathbf{K}-\Lambda\|_{L^{\infty}\left(I \times \mathbb{T}^{d}\right)} \lesssim N^{d-\frac{1}{2}}
$$

Hence, we have

$$
|\langle f, f *(\mathbf{K}-\Lambda)\rangle| \lesssim N^{d-\frac{1}{2}}\|f\|_{L^{1}}^{2} .
$$


Let $p \in(1,2)$ such that

$$
\frac{1}{p}=\frac{1-\theta}{1}+\frac{\theta}{2}
$$

for some $\theta \in(0,1)$. Note that $p^{\prime} \theta=2$.

Case (i): $\theta<\frac{1}{5}$.

By interpolating (3.33) and (3.39), we have

$$
\left\|f * \Lambda_{M, s}\right\|_{L^{p^{\prime}}\left(I \times \mathbb{T}^{d}\right)} \lesssim N^{(d-1)(1-\theta)}\left(2^{s} N\right)^{\frac{1}{2}-\frac{3}{2} \theta} M^{-\frac{1}{2}+\left(\frac{5}{2}+\right) \theta}\|f\|_{L^{p}\left(I \times \mathbb{T}^{d}\right)} .
$$

Then, summing over dyadic $M \geq 1$ and $s$ with $2^{s} \leq N$, we have

$$
\|f * \Lambda\|_{L^{p^{\prime}\left(I \times \mathbb{T}^{d}\right)}} \lesssim N^{d-(d+2) \theta}\|f\|_{L^{p}\left(I \times \mathbb{T}^{d}\right)},
$$

as long as $\theta>0$ satisfies $-\frac{1}{2}+\left(\frac{5}{2}+\right) \theta \leq 0$, i.e.

$$
\theta<\frac{1}{5}
$$

In the following, we prove the level set estimate (3.5) for $q>10$. Given $f$ as in (3.2), let $F(\mathbf{x}, t)=e^{-i t \Delta} f(\mathbf{x}, t)$. Let $\Theta_{\lambda}(\mathbf{x}, t)$ be as in (3.16), where $A_{\lambda}=\left\{(\mathbf{x}, t) \in \mathbb{T}^{d} \times I\right.$ : $|F(\mathbf{x}, t)|>\lambda\}$. Then, proceeding as in (3.17) with (3.49) and (3.52), we have

$$
\begin{aligned}
\lambda^{2}\left|A_{\lambda}\right|^{2} & \leq \sum_{\mathbf{n} \in S_{N}}\left|\widehat{\Theta}_{\lambda}(\mathbf{n}, Q(\mathbf{n}))\right|^{2} \leq\left\langle\mathbf{K} * \Theta_{\lambda}, \Theta_{\lambda}\right\rangle \\
& \leq\left|\left\langle(\mathbf{K}-\Lambda) * \Theta_{\lambda}, \Theta_{\lambda}\right\rangle\right|+\left|\left\langle\Lambda * \Theta_{\lambda}, \Theta_{\lambda}\right\rangle\right| \\
& \lesssim N^{d-\frac{1}{2}}\left|A_{\lambda}\right|^{2}+N^{d-(d+2) \theta}\left|A_{\lambda}\right|^{\frac{2}{p}} .
\end{aligned}
$$

For $\lambda \gg N^{\frac{d}{2}-\frac{1}{4}},(3.54)$ reduces to

$$
\lambda^{2}\left|A_{\lambda}\right|^{2} \lesssim N^{d-(d+2) \theta}\left|A_{\lambda}\right|^{\frac{2}{p}}
$$

Noting that $p^{\prime} \theta=2$ by (3.50), it follows from (3.55) that

$$
\left|A_{\lambda}\right| \lesssim N^{\frac{d}{2} q-(d+2)} \lambda^{-q}
$$

where $q:=p^{\prime}=\frac{2}{\theta}>10$. Note that we only needed to assume $\lambda \gg N^{\frac{d}{2}-\frac{1}{4}}$ and did not need the condition $\lambda \gtrsim N^{\frac{d}{2}-\varepsilon}$ in this case.

Case (ii): $\frac{1}{5} \leq \theta<\frac{1}{3}$.

Let $M_{j}, j=1,2$ be dyadic numbers such that

$$
M_{1} \sim\left(\frac{N^{\frac{d}{2}}}{\lambda}\right)^{\delta_{1}} \lesssim N^{\varepsilon \delta_{1}} \quad \text { and } \quad M_{2} \sim N^{\delta_{2}}
$$

Here, we choose $\delta_{1}, \delta_{2}>0$ such that $M_{1} \ll M_{2}$. We divide $\Lambda$ into three pieces: $\Lambda=$ $\Lambda_{1}+\Lambda_{2}+\Lambda_{3}$ by setting

$$
\Lambda_{j}=\sum_{\substack{M \in I_{j} \\ M, \text { dyadic }}} \sum_{M \leq 2^{s} \leq N} \Lambda_{M, s},
$$

where $I_{1}=\left[1, M_{1}\right], I_{2}=\left(M_{1}, M_{2}\right]$, and $I_{3}=\left(M_{2}, N_{1}\right]$ with $N_{1}=\frac{1}{100} N$ as before. 
Then, summing (3.51) over dyadic $M \leq M_{1}$ and $2^{s} \leq N$, we have

$$
\left\|f * \Lambda_{1}\right\|_{L^{p^{\prime}}\left(I \times \mathbb{T}^{d}\right)} \lesssim M_{1}^{-\frac{1}{2}+\left(\frac{5}{2}+\right) \theta} N^{d-(d+2) \theta}\|f\|_{L^{p}\left(I \times \mathbb{T}^{d}\right)},
$$

since $\theta \in\left[\frac{1}{5}, \frac{1}{3}\right)$.

Similarly, by interpolating (3.33) and (3.45), we have

$$
\begin{aligned}
\left\|f * \Lambda_{M, s}\right\|_{L^{p^{\prime}}\left(I \times \mathbb{T}^{d}\right)} \lesssim N^{(d-1)(1-\theta)}\left(2^{s} N\right)^{\frac{1}{2}-\frac{3}{2} \theta} M^{-\frac{1}{2}+\left(\frac{3}{2}+\right) \theta} D^{\theta}\|f\|_{L^{p}\left(I \times \mathbb{T}^{d}\right)} \\
\quad+N^{(d-1)(1-\theta)}\left(\frac{2^{s} N}{M}\right)^{\frac{1}{2}(1-\theta)} N^{\frac{d}{2} \theta}\left(\frac{D^{-B} M^{2+\beta+}}{2^{\frac{s}{2}} N^{\frac{1}{2}}}+\frac{M^{2+B+}}{2^{s} N}\right)^{\theta}\|f\|_{L^{1}\left(I \times \mathbb{T}^{d}\right)} .
\end{aligned}
$$

Now, choose $D \sim M^{\alpha}$ for some small $\alpha>0$. Then, set $\beta \ll 1$ and $B \gg 1$ such that

$$
\sigma:=-\frac{5}{2}-\beta+\alpha B->0
$$

Then, summing (3.59) over dyadic $M \in\left(M_{1}, M_{2}\right]$ and $s$ with $2^{s} \leq N$, we have

$$
\begin{aligned}
\left\|f * \Lambda_{2}\right\|_{L^{p^{\prime}}\left(I \times \mathbb{T}^{d}\right)} & \lesssim N^{d-(d+2) \theta}\|f\|_{L^{p}\left(I \times \mathbb{T}^{d}\right)} \\
& +\left(M_{1}^{-\frac{1}{2}-\sigma \theta} N^{d-\left(\frac{d}{2}+1\right) \theta}+M_{2}^{-\frac{1}{2}+\left(\frac{5}{2}+B+\right) \theta} N^{d-\left(\frac{d}{2}+2\right) \theta}\right)\|f\|_{L^{1}\left(I \times \mathbb{T}^{d}\right)},
\end{aligned}
$$

as long as $-\frac{1}{2}+\left(\frac{3}{2}+\alpha+\right) \theta \leq 0$, i.e.

$$
\theta<\frac{1}{3+2 \alpha+}
$$

Note that (3.62) can be satisfied as long as $\theta<\frac{1}{3}$ by choosing $\alpha$ sufficiently small.

Lastly, from (3.33) and (3.38), we have

$$
\left\|f * \Lambda_{M, s}\right\|_{L^{p^{\prime}}\left(I \times \mathbb{T}^{d}\right)} \lesssim N^{(d-1)(1-\theta)}\left(\frac{2^{s} N}{M}\right)^{\frac{1}{2}-\frac{3}{2} \theta} N^{(0+) \theta}\|f\|_{L^{p}\left(I \times \mathbb{T}^{d}\right)} .
$$

Then, summing over $M \geq M_{2} \sim N^{\delta_{2}}$ and $s$ with $2^{s} \leq N$, we have

$$
\begin{aligned}
\left\|f * \Lambda_{3}\right\|_{L^{p^{\prime}\left(I \times \mathbb{T}^{d}\right)}} & \lesssim N^{(d-1)(1-\theta)}\left(\frac{N^{2}}{M_{2}}\right)^{\frac{1}{2}-\frac{3}{2} \theta} N^{(0+) \theta}\|f\|_{L^{p}\left(I \times \mathbb{T}^{d}\right)} \\
& \lesssim N^{d-(d+2) \theta}\|f\|_{L^{p}\left(I \times \mathbb{T}^{d}\right)},
\end{aligned}
$$

as long as $\theta<\frac{1}{3}$.

Putting (3.58), (3.61), and (3.64) together with (3.53), we obtain

$$
\begin{aligned}
\|f * \Lambda\|_{L^{p^{\prime}}\left(I \times \mathbb{T}^{d}\right)} & \lesssim M_{1}^{-\frac{1}{2}+\left(\frac{5}{2}+\right) \theta} N^{d-(d+2) \theta}\|f\|_{L^{p}\left(I \times \mathbb{T}^{d}\right)} \\
& +\left(M_{1}^{-\frac{1}{2}-\sigma \theta} N^{d-\left(\frac{d}{2}+1\right) \theta}+M_{2}^{-\frac{1}{2}+\left(\frac{5}{2}+B+\right) \theta} N^{d-\left(\frac{d}{2}+2\right) \theta}\right)\|f\|_{L^{1}\left(I \times \mathbb{T}^{d}\right)} .
\end{aligned}
$$

Now, we are ready to prove the level set estimate (3.5) for $q>6$. Then, proceeding as in Case (i) with (3.49) and (3.65), we have

$$
\begin{aligned}
\lambda^{2}\left|A_{\lambda}\right|^{2} & \leq\left|\left\langle(\mathbf{K}-\Lambda) * \Theta_{\lambda}, \Theta_{\lambda}\right\rangle\right|+\left|\left\langle\Lambda * \Theta_{\lambda}, \Theta_{\lambda}\right\rangle\right| \\
& \lesssim N^{d-\frac{1}{2}}\left|A_{\lambda}\right|^{2}+M_{1}^{-\frac{1}{2}+\left(\frac{5}{2}+\right) \theta} N^{d-(d+2) \theta}\left|A_{\lambda}\right|^{\frac{2}{p}} \\
& \quad+M_{1}^{-\frac{1}{2}-\sigma \theta} N^{d-\left(\frac{d}{2}+1\right) \theta}\left|A_{\lambda}\right|^{1+\frac{1}{p}}+M_{2}^{-\frac{1}{2}+\left(\frac{5}{2}+B+\right) \theta} N^{d-\left(\frac{d}{2}+2\right) \theta}\left|A_{\lambda}\right|^{1+\frac{1}{p}}
\end{aligned}
$$


Since $\lambda \gg N^{\frac{d}{2}-\frac{1}{4}},(3.66)$ reduces to

$$
\begin{aligned}
\lambda^{2}\left|A_{\lambda}\right|^{2} \lesssim & M_{1}^{-\frac{1}{2}+\left(\frac{5}{2}+\right) \theta} N^{d-(d+2) \theta}\left|A_{\lambda}\right|^{\frac{2}{p}}+M_{1}^{-\frac{1}{2}-\sigma \theta} N^{d-\left(\frac{d}{2}+1\right) \theta}\left|A_{\lambda}\right|^{1+\frac{1}{p}} \\
& \quad+M_{2}^{-\frac{1}{2}+\left(\frac{5}{2}+B+\right) \theta} N^{d-\left(\frac{d}{2}+2\right) \theta}\left|A_{\lambda}\right|^{1+\frac{1}{p}} \\
= & \mathrm{I}+\mathrm{II}+\mathrm{III} .
\end{aligned}
$$

First, suppose that $\lambda^{2}\left|A_{\lambda}\right|^{2} \lesssim$ I holds. Recall from (3.50) that $p^{\prime} \theta=2$. Then, with (3.56), we have

$$
\left|A_{\lambda}\right| \lesssim\left(\frac{N^{\frac{d}{2}}}{\lambda}\right)^{\left(-\frac{p^{\prime}}{4}+\frac{5}{2}+\right) \delta_{1}} N^{\frac{d}{2} p^{\prime}-(d+2)} \lambda^{-p^{\prime}} \lesssim N^{\frac{d}{2} q-(d+2)} \lambda^{-q}
$$

for $q>p^{\prime}$ by choosing $\delta_{1}=\delta_{1}\left(q, p^{\prime}\right)$ sufficiently small.

Next, suppose that $\lambda^{2}\left|A_{\lambda}\right|^{2} \lesssim \Pi$ holds. Then, from (3.56), we have

$$
\left|A_{\lambda}\right| \lesssim N^{\frac{d}{2} p^{\prime}-(d+2)} \lambda^{-p^{\prime}}\left(N^{\frac{d}{2} p^{\prime}} \lambda^{-p^{\prime}} M_{1}^{-\frac{p^{\prime}}{2}-2 \sigma}\right) \lesssim N^{\frac{d}{2} p^{\prime}-(d+2)} \lambda^{-p^{\prime}}
$$

by making $\sigma=\sigma\left(p^{\prime}, \delta_{1}\right)$ in (3.60) (and hence $B=B\left(p^{\prime}, \delta_{1}\right)$ ) sufficiently large.

Lastly, suppose that $\lambda^{2}\left|A_{\lambda}\right|^{2} \lesssim \mathrm{III}$ holds. By $\lambda \gtrsim N^{\frac{d}{2}-\varepsilon}$ and (3.56), we have

$$
\left|A_{\lambda}\right| \lesssim N^{\frac{d}{2} p^{\prime}-(d+2)} \lambda^{-p^{\prime}} N^{-2+\varepsilon p^{\prime}+\left(-\frac{p^{\prime}}{2}+5+2 B+\right) \delta_{2}} \lesssim N^{\frac{d}{2} p^{\prime}-(d+2)} \lambda^{-p^{\prime}}
$$

as long as we have $\varepsilon p^{\prime} \leq 1$ and $\delta_{2}=\delta_{2}\left(p^{\prime}, B\right)$ is sufficiently small such that $\left(-\frac{p^{\prime}}{2}+5+\right.$ $2 B+) \delta_{2} \leq 1$

Finally, given $q>6$, we choose $\theta<\frac{1}{3}$ such that $q>p^{\prime}=\frac{2}{\theta}>6$. Then, from (3.67), (3.68), (3.69), and (3.70) with $\lambda \leq N^{\frac{d}{2}}$, we obtain

$$
\left|A_{\lambda}\right| \lesssim N^{\frac{d}{2} q-(d+2)} \lambda^{-q}
$$

This completes the proof of Proposition 3.1(ii).

\section{WELL-POSEDNESS IN SUBCRITICAL SPACES}

In this section, we prove local well-posedness of NLS (1.25) on irrational tori in subcritical Sobolev spaces (Theorem 1.4). It turns out that the well-posed theory of (1.25) is very similar to that on the standard tours [3, 4, 5, 6].

In the seminal paper [3], Bourgain introduced the $X^{s, b}$-space whose norm is given by

$$
\|u\|_{X^{s, b}\left(\mathbb{R} \times \mathbb{T}^{d}\right)}=\left\|\langle\mathbf{n}\rangle^{s}\left\langle\tau-|\mathbf{n}|^{2}\right\rangle^{b} \widehat{u}(\mathbf{n}, \tau)\right\|_{L_{\tau}^{2} \ell_{\mathbf{n}}^{2}\left(\mathbb{R} \times \mathbb{Z}^{d}\right)},
$$

where $\langle\cdot\rangle=\left(1+|\cdot|^{2}\right)^{\frac{1}{2}}$. After establishing Strichartz estimates, he proved several wellposedness results of NLS on the standard torus $\mathbb{T}^{d}$. In our case, i.e. on an irrational torus, we need to replace the weight $\left\langle\tau-|\mathbf{n}|^{2}\right\rangle^{b}$ in (4.1) by $\langle\tau-Q(\mathbf{n})\rangle^{b}$, where $Q(\mathbf{n})$ is defined in (1.15). Then, by the standard $X^{s, b}$-theory, it is known that certain multilinear Strichartz estimates imply well-posedness. More precisely, we have the following lemma. 
Lemma 4.1. Let $s_{0}>\max \left(0, s_{c}\right)$ and $I \subset \mathbb{R}$ be a bounded interval. Suppose that the following multilinear Strichartz estimate holds for $s>s_{0}$ :

$$
\left\|\prod_{j=1}^{k+1} e^{-i t \Delta} \phi_{j}\right\|_{L_{t, \mathbf{x}}^{2}\left(I \times \mathbb{T}^{d}\right)} \lesssim N_{\max }^{-s} \prod_{j=1}^{k+1} N_{j}^{s}\left\|\phi_{i}\right\|_{L_{\mathbf{x}}^{2}\left(\mathbb{T}^{d}\right)}
$$

for all $\phi_{j} \in L^{2}\left(\mathbb{T}^{d}\right)$ with $\operatorname{supp} \widehat{\phi}_{j} \subset\left[-N_{j}, N_{j}\right]^{d}, j=1, \ldots, k+1$, and $N_{\max }:=$ $\max \left(N_{1}, \cdots, N_{k+1}\right)$. Then, the Cauchy problem (1.25) is locally well-posed in $H^{s}\left(\mathbb{T}^{d}\right)$ for $s>s_{0}$.

The proof of Lemma 4.1 is standard and we refer the readers to [3, 4, 6, 11] for details.

Proof of Theorem 1.4. In view of Lemma 4.1, it suffices to prove the $(k+1)$-linear estimate (4.2) for $s>s_{0}$. Without loss of generality, assume that $N_{1} \geq N_{2} \geq \cdots \geq N_{k+1}$.

Case (i): $d=2$. When $k=1$, the well-posedness result was already obtain in [11]. In the following, we first consider the case $k=2,3,4,5$. First, assume that $\operatorname{supp} \widehat{\phi}_{1} \subset\left[-N_{2}, N_{2}\right]^{2}$. Then, by Hölder's inequality and (1.21) with $\frac{4(7 k+5)}{3(k+3)} \in\left(4, \frac{20}{3}\right]$, we have

$$
\begin{aligned}
\left\|\prod_{j=1}^{k+1} e^{-i t \Delta} \phi_{j}\right\|_{L_{t, \mathbf{x}}^{2}} & \leq\left\|e^{-i t \Delta} \phi_{1}\right\|_{L^{\frac{4(7 k+5)}{3(k+3)}}}\left\|e^{-i t \Delta} \phi_{2}\right\|_{L^{\frac{4(7 k+5)}{3(k+3)}}} \prod_{j=3}^{k+1}\left\|e^{-i t \Delta} \phi_{j}\right\|_{L^{\frac{7 k+5}{2}}} \\
& \lesssim N_{2}^{\frac{7 k-3}{7 k+5}+2 \varepsilon}\left\|\phi_{1}\right\|_{L^{2}}\left\|\phi_{2}\right\|_{L^{2}} \prod_{j=3}^{k+1} N_{j}^{\frac{7 k-3}{7 k+5}+\varepsilon}\left\|\phi_{j}\right\|_{L^{2}} \\
& \leq N_{\max }^{-s} \prod_{j=1}^{k+1} N_{j}^{s}\left\|\phi_{i}\right\|_{L_{\mathbf{x}}^{2}}
\end{aligned}
$$

for $s>s_{0}=\frac{7 k-3}{7 k+5}$. In general, if $\operatorname{supp} \widehat{\phi}_{1} \subset\left[-N_{1}, N_{1}\right]^{2}$, then we can write $\phi_{1}=\sum_{|\mathbf{j}| \lesssim \frac{N_{1}}{N_{2}}} \phi_{1 \mathbf{j}}$, where supp $\widehat{\phi}_{1 \mathbf{j}} \subset N_{2} \mathbf{j}+\left[-N_{2}, N_{2}\right]^{2}$. Letting $\psi_{1 \mathbf{j}}(\mathbf{x})=e^{-2 \pi i N_{2} \mathbf{j} \cdot \mathbf{x}} \phi_{1 \mathbf{j}}(\mathbf{x})$, we have supp $\widehat{\psi}_{1 \mathbf{j}} \subset$ $\left[-N_{2}, N_{2}\right]^{2}$. Then, by a change of variables and (1.21) (see [5]), we obtain

$$
\left\|e^{-i t \Delta} \phi_{1 \mathbf{j}}\right\|_{L_{t, \mathbf{x}}}^{\frac{4(7 k+3)}{3(k+3)}}=\left\|e^{-i t \Delta} \psi_{1 \mathbf{j}}\right\|_{L^{\frac{4(7 k+5)}{3(k+3)}}} \lesssim N_{2}^{\frac{7 k-3}{2(7 k+5)}+\varepsilon}\left\|\phi_{1 \mathbf{j}}\right\|_{L_{\mathbf{x}}^{2}} .
$$

Then, by almost orthogonality with (4.3) and (4.4), we have

$$
\left\|\prod_{j=1}^{k+1} e^{-i t \Delta} \phi_{j}\right\|_{L_{t, \mathbf{x}}^{2}}^{2} \lesssim \sum_{|\mathbf{j}| \lesssim \frac{N_{1}}{N_{2}}}\left\|e^{-i t \Delta} \phi_{1 \mathbf{j}} \prod_{j=2}^{k+1} e^{i t \Delta} \phi_{j}\right\|_{L_{t, \mathbf{x}}^{2}}^{2} \lesssim N_{\max }^{-2 s} \prod_{j=1}^{k+1} N_{j}^{2 s}\left\|\phi_{j}\right\|_{L_{\mathbf{x}}^{2}}^{2} .
$$

Remark 4.2. In view of the reduction above, we only prove (4.2), assuming supp $\widehat{\phi}_{1} \subset$ $\left[-N_{2}, N_{2}\right]^{d}$ in the following. 
Next, we consider the case $k \geq 5$. By Hölder's inequality and (1.21) with $\frac{8 k}{k+1} \in\left[\frac{20}{3}, 10\right)$, we have

$$
\begin{aligned}
\left\|\prod_{j=1}^{k+1} e^{-i t \Delta} \phi_{j}\right\|_{L_{t, \mathbf{x}}^{2}} & \leq\left\|e^{-i t \Delta} \phi_{1}\right\|_{L^{\frac{8 k}{k+1}}}\left\|e^{-i t \Delta} \phi_{2}\right\|_{L^{\frac{8 k}{k+1}}} \prod_{j=3}^{k+1}\left\|e^{-i t \Delta} \phi_{j}\right\|_{L^{4} k} \\
& \lesssim N_{2}^{1-\frac{1}{k}+2 \varepsilon}\left\|\phi_{1}\right\|_{L^{2}}\left\|\phi_{2}\right\|_{L^{2}} \prod_{j=3}^{k+1} N_{j}^{1-\frac{1}{k}}\left\|\phi_{j}\right\|_{L_{\mathbf{x}}^{2}}
\end{aligned}
$$

Hence, (4.2) holds for $s>s_{0}=1-\frac{1}{k}$.

Case (ii): $d=3$. When $k=1$, the well-posedness result was obtained in [6]. When $k=2$, by Hölder's inequality and (1.22), we have

$$
\begin{aligned}
\left\|\prod_{j=1}^{3} e^{-i t \Delta} \phi_{j}\right\|_{L_{t, \mathbf{x}}^{2}} & \leq\left\|e^{-i t \Delta} \phi_{1}\right\|_{L^{\frac{104}{21}}}\left\|e^{-i t \Delta} \phi_{2}\right\|_{L^{\frac{104}{21}}}\left\|e^{-i t \Delta} \phi_{3}\right\|_{L^{\frac{52}{5}}} \\
& \lesssim N_{2}^{\frac{53}{52}+2 \varepsilon} N_{3}^{\frac{53}{52}} \prod_{j=1}^{3}\left\|\phi_{j}\right\|_{L_{\mathbf{x}}^{2}}
\end{aligned}
$$

Hence, (4.2) holds for $s>s_{0}=\frac{53}{52}$. When $k \geq 3$, by Hölder's inequality and (1.22), we have

$$
\begin{aligned}
\left\|\prod_{j=1}^{k+1} e^{-i t \Delta} \phi_{j}\right\|_{L_{t, \mathbf{x}}^{2}} & \leq\left\|e^{-i t \Delta} \phi_{1}\right\|_{L^{\frac{20 k}{3 k+2}}}\left\|e^{-i t \Delta} \phi_{2}\right\|_{L^{\frac{20 k}{3 k+2}}} \prod_{j=3}^{k+1}\left\|e^{-i t \Delta} \phi_{j}\right\|_{L^{5 k}} \\
& \lesssim N_{2}^{\frac{3}{2}-\frac{1}{k}}\left\|\phi_{1}\right\|_{L^{2}}\left\|\phi_{2}\right\|_{L^{2}} \prod_{j=3}^{k+1} N_{j}^{\frac{3}{2}-\frac{1}{k}}\left\|\phi_{j}\right\|_{L_{\mathbf{x}}^{2}}
\end{aligned}
$$

Hence, (4.2) holds $s \geq s_{0}=\frac{3}{2}-\frac{1}{k}$.

Case (iii): $d \geq 4$ and $k \geq 1$. By Hölder's inequality with (1.23) or (1.24), we have

$$
\begin{aligned}
\left\|\prod_{j=1}^{k+1} e^{-i t \Delta} \phi_{j}\right\|_{L_{t, \mathbf{x}}^{2}} & \leq\left\|e^{-i t \Delta} \phi_{1}\right\|_{L^{\frac{4(d+2) k}{d k+2}}}\left\|e^{-i t \Delta} \phi_{2}\right\|_{L^{\frac{4(d+2) k}{d k+2}}} \prod_{j=3}^{k+1}\left\|e^{-i t \Delta} \phi_{j}\right\|_{L^{(d+2) k}} \\
& \lesssim N_{2}^{\frac{d}{2}-\frac{1}{k}+\varepsilon}\left\|\phi_{1}\right\|_{L^{2}}\left\|\phi_{2}\right\|_{L^{2}} \prod_{j=3}^{k+1} N_{j}^{\frac{d}{2}-\frac{1}{k}}\left\|\phi_{j}\right\|_{L_{\mathbf{x}}^{2}} .
\end{aligned}
$$

Hence, (4.2) holds for $s>s_{0}=\frac{d}{2}-\frac{1}{k}$.

\section{WELL-POSEDNESS IN CRITICAL SPACES}

5.1. Function spaces. In this section, we prove local well-posedness of NLS (1.25) on irrational tori in critical Sobolev spaces $H^{s_{c}}\left(\mathbb{T}^{d}\right)$ (Theorem 1.5). In the following, we use the $U^{p}$ - and $V^{p}$-spaces, developed by Tataru, Koch, and their collaborators [30, 18, 21, 22]. These spaces have been very effective in establishing well-posedness of various dispersive PDEs in critical spaces. We briefly go over the basic definitions of function spaces and their properties. See Hadac-Herr-Koch [18] and Herr-Tataru-Tzvetkov [21] for detailed proofs. 
Let $H$ be a separable Hilbert space over $\mathbb{C}$. In particular, it will be either $H^{s}\left(\mathbb{T}^{d}\right)$ or $\mathbb{C}$. Let $\mathcal{Z}$ be the collection of finite partitions $\left\{t_{k}\right\}_{k=0}^{K}$ of $\mathbb{R}:-\infty<t_{0}<\cdots<t_{K} \leq \infty$. If $t_{K}=\infty$, we use the convention $u\left(t_{K}\right):=0$ for all functions $u: \mathbb{R} \rightarrow H$. We use $\mathbf{1}_{I}$ to denote the sharp characteristic function of a set $I \subset \mathbb{R}$.

Definition 5.1. Let $1 \leq p<\infty$.

(i) A $U^{p}$-atom is defined by a step function $a: \mathbb{R} \rightarrow H$ of the form

$$
a=\sum_{k=1}^{K} \mathbf{1}_{\left[t_{k-1}, t_{k}\right)} \phi_{k-1}
$$

where $\left\{t_{k}\right\}_{k=0}^{K} \in \mathcal{Z}$ and $\left\{\phi_{k}\right\}_{k=0}^{K-1} \subset H$ with $\sum_{k=0}^{K-1}\left\|\phi_{k}\right\|_{H}^{p}=1$. Then, we define the atomic space $U^{p}(\mathbb{R} ; H)$ to be the collection of functions $u: \mathbb{R} \rightarrow H$ of the form

$$
u=\sum_{j=1}^{\infty} \lambda_{j} a_{j}, \quad \text { where } a_{j} \text { 's are } U^{p} \text {-atoms and }\left\{\lambda_{j}\right\}_{j \in \mathbb{N}} \in \ell^{1}(\mathbb{N} ; \mathbb{C}),
$$

with the norm

$$
\|u\|_{U^{p}(\mathbb{R} ; H)}:=\inf \left\{\|\lambda\|_{\ell^{1}}: \text { (5.1) holds with } \lambda=\left\{\lambda_{j}\right\}_{j \in \mathbb{N}} \text { and some } U^{p} \text {-atoms } a_{j}\right\} .
$$

(ii) We define $V^{p}(\mathbb{R} ; H)$ by the collection of functions $u: \mathbb{R} \rightarrow H$ with $\|u\|_{V^{p}(\mathbb{R} ; H)}<\infty$, where the $V^{p}$-norm is defined by

$$
\|u\|_{V^{p}(\mathbb{R} ; H)}:=\sup _{\left\{t_{k}\right\}_{k=0}^{K} \in \mathcal{Z}}\left(\sum_{k=1}^{K}\left\|u\left(t_{k}\right)-u\left(t_{k-1}\right)\right\|_{H}^{p}\right)^{\frac{1}{p}} .
$$

We also define $V_{\mathrm{rc}}^{p}(\mathbb{R} ; H)$ to be the closed subspace of all right-continuous functions in $V^{p}(\mathbb{R} ; H)$ such that $\lim _{t \rightarrow-\infty} u(t)=0$.

(iii) Let $s \in \mathbb{R}$. We define $U_{\Delta}^{p} H^{s}$ (and $V_{\Delta}^{p} H^{s}$, respectively) to be the spaces of all functions $u: \mathbb{R} \rightarrow H^{s}\left(\mathbb{T}^{d}\right)$ such that the following $U_{\Delta}^{p} H^{s}$-norm (and $V_{\Delta}^{p} H^{s}$-norm, respectively) is finite:

$$
\|u\|_{U_{\Delta}^{p} H^{s}}:=\left\|e^{i t \Delta} u\right\|_{U^{p}\left(\mathbb{R} ; H^{s}\right)} \quad \text { and } \quad\|u\|_{V_{\Delta}^{p} H^{s}}:=\left\|e^{i t \Delta} u\right\|_{V^{p}\left(\mathbb{R} ; H^{s}\right)}
$$

Here, the Laplacian $\Delta$ is defined in terms of $Q(\mathbf{n})$ as in (1.14).

Remark 5.2. Note that the spaces $U^{p}(\mathbb{R} ; H), V^{p}(\mathbb{R} ; H)$, and $V_{\mathrm{rc}}^{p}(\mathbb{R} ; H)$ are Banach spaces. Moreover, we have the following embeddings:

$$
U^{p}(\mathbb{R} ; H) \hookrightarrow V_{\mathrm{rc}}^{p}(\mathbb{R} ; H) \hookrightarrow U^{q}(\mathbb{R} ; H) \hookrightarrow L^{\infty}(\mathbb{R} ; H)
$$

for $1 \leq p<q<\infty$. Similar embeddings hold for $U_{\Delta}^{p} H^{s}$ and $V_{\Delta}^{p} H^{s}$.

Next, we state a transference principle and an interpolation result.

Lemma 5.3. (i) (transference principle) Suppose that we have

$$
\left\|T\left(e^{-i t \Delta} \phi_{1}, \ldots, e^{-i t \Delta} \phi_{k}\right)\right\|_{L_{t}^{p} L_{\mathbf{x}}^{q}\left(\mathbb{R} \times \mathbb{T}^{d}\right)} \lesssim \prod_{j=1}^{k}\left\|\phi_{j}\right\|_{L_{\mathbf{x}}^{2}}
$$


for some $1 \leq p, q \leq \infty$. Then, we have

$$
\left\|T\left(u_{1}, \ldots, u_{k}\right)\right\|_{L_{t}^{p} L_{\mathbf{x}}^{q}\left(\mathbb{R} \times \mathbb{T}^{d}\right)} \lesssim \prod_{j=1}^{k}\left\|u_{j}\right\|_{U_{\Delta}^{p} L_{\mathbf{x}}^{2}} .
$$

(ii) (interpolation) Let $E$ be a Banach space. Suppose that $T: U^{p_{1}} \times \cdots \times U^{p_{k}} \rightarrow E$ is a bounded k-linear operator such that

$$
\left\|T\left(u_{1}, \ldots, u_{k}\right)\right\|_{E} \leq C_{1} \prod_{j=1}^{k}\left\|u_{j}\right\|_{U^{p_{j}}}
$$

for some $p_{1}, \ldots, p_{k}>2$. Moreover, assume that there exists $C_{2} \in\left(0, C_{1}\right]$ such that

$$
\left\|T\left(u_{1}, \ldots, u_{k}\right)\right\|_{E} \leq C_{2} \prod_{j=1}^{k}\left\|u_{j}\right\|_{U^{2}} .
$$

Then, we have

$$
\left\|T\left(u_{1}, \ldots, u_{k}\right)\right\|_{E} \leq C_{2}\left(\ln \frac{C_{1}}{C_{2}}+1\right)^{k} \prod_{j=1}^{k}\left\|u_{j}\right\|_{V^{2}}
$$

for $u_{j} \in V_{\mathrm{rc}}^{2}, j=1, \ldots, k$.

A transference principle as above has been commonly used in the Fourier restriction norm method. See [18, Proposition 2.19] for the proof of Lemma 5.3 (i). The proof of the interpolation result follows from extending the trilinear result in [21] to a general $k$-linear case. See also [18, Proposition 2.20].

Let $\eta: \mathbb{R} \rightarrow[0,1]$ be an even smooth cutoff function supported on $\left[-\frac{8}{5}, \frac{8}{5}\right]$ such that $\eta \equiv 1$ on $\left[-\frac{5}{4}, \frac{5}{4}\right]$. Given a dyadic number $N \geq 1$, we set $\eta_{1}(\xi)=\eta(|\xi|)$ and

$$
\eta_{N}(\xi)=\eta\left(\frac{|\xi|}{N}\right)-\eta\left(\frac{2|\xi|}{N}\right)
$$

for $N \geq 2$. Then, we define the Littlewood-Paley projection operator $P_{N}$ as the Fourier multiplier operator with symbol $\eta_{N}$. Moreover, we define $P_{\leq N}$ by $P_{\leq N}=\sum_{1 \leq M \leq N} P_{M}$. More generally, given a set $R \subset \mathbb{Z}^{d}$, we define $P_{R}$ to be the Fourier multiplier operator with symbol $\mathbf{1}_{R}$.

Definition 5.4. (i) Let $s \in \mathbb{R}$. We define $X^{s}$ to be the space of all functions $u: \mathbb{R} \rightarrow H^{s}\left(\mathbb{T}^{d}\right)$ such that $\|u\|_{X^{s}}<\infty$, where the $X^{s}$-norm is defined by

$$
\|u\|_{X^{s}}:=\left(\sum_{\mathbf{n} \in \mathbb{Z}^{d}}\langle\mathbf{n}\rangle^{2 s}\left\|e^{-i t Q(\mathbf{n})} \widehat{u}(\mathbf{n}, t)\right\|_{U^{2}\left(\mathbb{R}_{t} ; \mathbb{C}\right)}^{2}\right)^{\frac{1}{2}} .
$$

(ii) Let $s \in \mathbb{R}$. We define $Y^{s}$ to be the space of all functions $u: \mathbb{R} \rightarrow H^{s}\left(\mathbb{T}^{d}\right)$ such that for every $\mathbf{n} \in \mathbb{Z}^{d}$, the map $t \mapsto e^{-i t Q(\mathbf{n})} \widehat{u}(\mathbf{n}, t)$ is in $V_{\mathrm{rc}}^{2}\left(\mathbb{R}_{t} ; \mathbb{C}\right)$ and $\|u\|_{Y^{s}}<\infty$, where the $Y^{s}$-norm is defined by

$$
\|u\|_{Y^{s}}:=\left(\sum_{\mathbf{n} \in \mathbb{Z}^{d}}\langle\mathbf{n}\rangle^{2 s}\left\|e^{-i t Q(\mathbf{n})} \widehat{u}(\mathbf{n}, t)\right\|_{V^{2}\left(\mathbb{R}_{t} ; \mathbb{C}\right)}^{2}\right)^{\frac{1}{2}} .
$$


Recall the following embeddings:

$$
U_{\Delta}^{2} H^{s} \hookrightarrow X^{s} \hookrightarrow Y^{s} \hookrightarrow V_{\Delta}^{2} H^{s} .
$$

Given a time interval $I \subset \mathbb{R}$, we define the restrictions $X^{s}(I)$ and $Y^{s}(I)$ of these spaces in the usual manner.

We now state the linear estimates. Given $f \in L_{\text {loc }}^{1}\left([0, \infty) ; L^{2}\left(\mathbb{T}^{d}\right)\right)$, define $\mathcal{I}(f)$ by

$$
\mathcal{I}(f)(t):=\int_{0}^{t} e^{-i\left(t-t^{\prime}\right) \Delta} f\left(t^{\prime}\right) d t^{\prime} .
$$

Lemma 5.5 (Linear estimates). Let $s \geq 0$ and $T>0$. Then, the following linear estimates hold:

$$
\begin{aligned}
\left\|e^{-i t \Delta} \phi\right\|_{X^{s}([0, T))} & \leq\|\phi\|_{H^{s}}, \\
\|\mathcal{I}(f)\|_{X^{s}([0, T))} & \leq \sup _{\substack{v \in Y^{-s}([0, T)) \\
\|v\|_{Y^{-s}=1}}}\left|\int_{0}^{T} \int_{\mathbb{T}^{d}} f(\mathbf{x}, t) \overline{v(\mathbf{x}, t)} d \mathbf{x} d t\right|,
\end{aligned}
$$

for all $\phi \in H^{s}\left(\mathbb{T}^{d}\right)$ and $f \in L^{1}\left([0, T) ; H^{s}\left(\mathbb{T}^{d}\right)\right)$.

Next, we present the crucial multilinear estimate.

Proposition 5.6. Let $d$ and $k$ satisfy

$$
\text { (i) } d=2, \quad k \geq 6, \quad \text { (ii) } d=3, \quad k \geq 3, \quad \text { or } \quad \text { (iii) } d \geq 4, \quad k \geq 2 \text {. }
$$

Then, the following multilinear estimate holds for all $T \in(0,1]$ :

$$
\left\|\mathcal{I}\left(\prod_{j=1}^{2 k+1} u_{j}^{*}\right)\right\|_{X^{s_{c}([0, T))}} \lesssim \sum_{j=1}^{2 k+1}\left(\left\|u_{j}\right\|_{X^{s}([0, T))} \prod_{\substack{\ell=1 \\ \ell \neq j}}^{2 k+1}\left\|u_{\ell}\right\|_{X^{s_{c}([0, T))}}\right),
$$

for $s \geq s_{c}=\frac{d}{2}-\frac{1}{k}>0$, where $u_{j}^{*}$ denotes either $u_{j}$ or $\bar{u}_{j}$.

Once we prove Proposition [5.6, one can prove Theorem 1.5 by the fixed point argument as in [21, 42]. We omit details. The remainder of this section is devoted to the proof of Proposition [5.6. Indeed, the multilinear estimate (5.4) follows once we prove the following multilinear Strichartz estimate.

Proposition 5.7. Let $d$ and $k$ satisfy (5.3). Then, there exists $\delta>0$ such that the following multilinear Strichartz estimate holds:

$$
\left\|\prod_{j=1}^{k+1} P_{N_{j}} e^{-i t \Delta} \phi_{j}\right\|_{L_{t, \mathbf{x}}^{2}\left(I \times \mathbb{T}^{d}\right)} \lesssim\left(\frac{N_{k+1}}{N_{1}}+\frac{1}{N_{2}}\right)^{\delta}\left\|P_{N_{1}} \phi_{1}\right\|_{L_{\mathbf{x}}^{2}\left(\mathbb{T}^{d}\right)} \prod_{j=2}^{k+1} N_{j}^{s_{c}}\left\|P_{N_{j}} \phi_{j}\right\|_{L_{\mathbf{x}}^{2}\left(\mathbb{T}^{d}\right)},
$$

for any interval $I \subset[0,1]$ and for all $\phi_{j} \in L^{2}\left(\mathbb{T}^{d}\right), j=1, \ldots, k+1$, and $N_{1} \geq N_{2} \geq \cdots \geq$ $N_{k+1} \geq 1$.

In Subsection 5.2, we first present the proof of Proposition 5.7. In Subsection 5.3, we then use the multilinear Strichartz estimate (5.5) to prove Proposition [5.6, thus yielding Theorem 1.5. 
5.2. Multilinear Strichartz estimate. In this subsection, we use the sharp $L^{p}$-Strichartz estimates (1.19) in Theorem 1.2 to prove the multilinear Strichartz estimate (5.5). The main idea is to refine the Strichartz estimate by considering frequency scales finer than the standard dyadic Littlewood-Paley localizations as in [21. See Lemma 5.10.

Definition 5.8. We say that $(d, p) \in \mathbb{N} \times \mathbb{R}$ is an admissible pair if

$$
\text { (i) } d=2, p>\frac{20}{3}, \quad \text { (ii) } d=3, p>\frac{16}{3}, \quad \text { or } \quad \text { (iii) } d \geq 4, p>4 \text {. }
$$

Note that, by Theorem 1.2, the Strichartz estimates with $(d, p)$ in this range are sharp.

Given dyadic $N \geq 1$, let $\mathcal{C}_{N}$ denote the collection of cubes $C \subset \mathbb{Z}^{d}$ of side length $\sim N$ with arbitrary center and orientation. Then, we can rewrite Theorem 1.2 in the following form.

Lemma 5.9. Let $(d, p)$ be admissible and $I \subset \mathbb{R}$ be a bounded interval. Then, for all dyadic $N \geq 1$, we have

$$
\left\|P_{N} e^{-i t \Delta} \phi\right\|_{L_{t, \mathbf{x}}^{p}\left(I \times \mathbb{T}^{d}\right)} \lesssim N^{\frac{d}{2}-\frac{d+2}{p}}\left\|P_{N} \phi\right\|_{L_{\mathbf{x}}^{2}\left(\mathbb{T}^{d}\right)} .
$$

More generally, for all $C \in \mathcal{C}_{N}$, we have

$$
\left\|P_{C} e^{-i t \Delta} \phi\right\|_{L_{t, \mathbf{x}}^{p}\left(I \times \mathbb{T}^{d}\right)} \lesssim N^{\frac{d}{2}-\frac{d+2}{p}}\left\|P_{C} \phi\right\|_{L_{\mathbf{x}}^{2}\left(\mathbb{T}^{d}\right)} .
$$

The Strichartz estimate (5.8) shows that the loss in (5.8) depends only on the size of the frequency support, not the position. See [5, 21].

In order to exploit further orthogonality between different frequency pieces under the linear Schrödinger evolution, we need to decompose the frequency cubes $C_{N}$. Let $\mathcal{R}_{M}(N)$ be the collection of all sets in $\mathbb{Z}^{d}$ which are given as the intersection of a cube of side length $2 N$ with a strip of "width" $2 M$, i.e. the collection of all sets of the form

$$
\left(\mathbf{n}_{0}+[-N, N]^{d}\right) \cap\left\{\mathbf{n} \in \mathbb{Z}^{d}:|\mathbf{a} \cdot \boldsymbol{\theta} \mathbf{n}-A| \leq M\right\}
$$

with some $\mathbf{n}_{0} \in \mathbb{Z}^{d}, \mathbf{a} \in \mathbb{R}^{d},|\mathbf{a}|=1, A \in \mathbb{R}$. Here, the $\operatorname{dot}$ product $\mathbf{a} \cdot \boldsymbol{\theta} \mathbf{n}$ is given by

$$
\mathbf{a} \cdot \boldsymbol{\theta} \mathbf{n}=\sum_{j=1}^{d} \theta_{j} a_{j} n_{j},
$$

where $\boldsymbol{\theta}=\left(\theta_{1}, \ldots, \theta_{d}\right)$ is as in (1.15). Then, we have the following refinement of the Strichartz estimate.

Lemma 5.10. Let $(d, p)$ be admissible and $I \subset \mathbb{R}$ be a bounded interval. Then, for all $1 \leq M \leq N$ and $R \in \mathcal{R}_{M}(N)$, we have

$$
\left\|P_{R} e^{-i t \Delta} \phi\right\|_{L_{t, \mathbf{x}}^{p}\left(I \times \mathbb{T}^{d}\right)} \lesssim N^{\frac{d}{2}-\frac{d+2}{p}}\left(\frac{M}{N}\right)^{\delta}\left\|P_{R} \phi\right\|_{L_{\mathbf{x}}^{2}\left(\mathbb{T}^{d}\right)},
$$

where $0<\delta<\frac{1}{2}-\frac{10}{3 p}$ when $d=2,0<\delta<\frac{1}{2}-\frac{8}{3 p}$ when $d=3$, and $0<\delta<\frac{1}{2}-\frac{2}{p}$ when $d \geq 4$. 
Proof. By Bernstein's inequality, we have

$$
\left\|P_{R} e^{-i t \Delta} \phi\right\|_{L_{t, \mathbf{x}}^{\infty}\left(I \times \mathbb{T}^{d}\right)} \lesssim M^{\frac{1}{2}} N^{\frac{d-1}{2}}\left\|P_{R} \phi\right\|_{L_{\mathbf{x}}^{2}\left(\mathbb{T}^{d}\right)} .
$$

Given admissible $(d, p)$, write $\frac{1}{p}=\frac{\theta}{q}+\frac{1-\theta}{\infty}$ for some $\theta \in[0,1)$, where $q<p$ is given by $q=\frac{20}{3}+$ when $d=2, q=\frac{16}{3}+$ when $d=3$, and $q=4+$ when $d \geq 4$. Then, (5.11) follows from interpolating (5.8) (with $p=q$ ) and (5.12).

Now, we are ready to prove the main multilinear Strichartz estimates (5.5).

Proof of Proposition 5.7. Let $u_{j}=e^{-i t \Delta} \phi_{j}$. Then, by almost orthogonality in spatial frequencies, it suffices to prove that there exists $\delta>0$ such that

$$
\left\|P_{C} P_{N_{1}} u_{1} \prod_{j=2}^{k+1} P_{N_{j}} u_{j}\right\|_{L_{t, \mathbf{x}}^{2}} \lesssim\left(\frac{N_{k+1}}{N_{1}}+\frac{1}{N_{2}}\right)^{\delta}\left\|P_{C} P_{N_{1}} \phi_{1}\right\|_{L_{\mathbf{x}}^{2}} \prod_{j=2}^{k+1} N_{j}^{s_{c}}\left\|P_{N_{j}} \phi_{j}\right\|_{L_{\mathbf{x}}^{2}},
$$

for all cubes $C \in \mathcal{C}_{N_{2}}$. Fix a cube $C \in \mathcal{C}_{N_{2}}$ and let $\mathbf{n}_{0}$ be the center of $C$. Partition $C=\bigcup R_{\ell}$ into disjoint strips $R_{\ell}$ with width $M=\max \left(N_{2}^{2} / N_{1}, 1\right)$, which are all orthogonal to $\mathbf{n}_{0}$ with respect to the dot product $\cdot \boldsymbol{\theta}$ in (5.10), i.e. $R_{\ell}$ is given by

$$
R_{\ell}=\left\{\mathbf{n} \in C: \mathbf{n} \cdot \boldsymbol{\theta} \mathbf{n}_{0} \in\left[\left|\mathbf{n}_{0}\right| M \ell,\left|\mathbf{n}_{0}\right| M(\ell+1)\right)\right\}, \quad|\ell| \sim \frac{N_{1}}{M} .
$$

Note that we have $R_{\ell} \in \mathcal{R}_{M}\left(N_{2}\right)$. By writing

$$
P_{C} P_{N_{1}} u_{1} \prod_{j=2}^{k+1} P_{N_{j}} u_{j}=\sum_{\ell} P_{R_{\ell}} P_{N_{1}} u_{1} \prod_{j=2}^{k+1} P_{N_{j}} u_{j}
$$

we show that the sum is almost orthogonal in $L_{t, \mathbf{x}}^{2}$. Since $N_{2}^{2} \lesssim M^{2} \ell$, we have

$$
Q\left(\mathbf{n}_{1}\right)=\frac{1}{Q\left(\mathbf{n}_{0}\right)}\left|\mathbf{n}_{1} \cdot \boldsymbol{\theta} \mathbf{n}_{0}\right|^{2}+Q\left(\mathbf{n}_{1}-\mathbf{n}_{0}\right)-\frac{1}{Q\left(\mathbf{n}_{0}\right)}\left|\left(\mathbf{n}_{1}-\mathbf{n}_{0}\right) \cdot \boldsymbol{\theta} \mathbf{n}_{0}\right|^{2}=M^{2} \ell^{2}+O\left(M^{2} \ell\right),
$$

for $\mathbf{n}_{1} \in R_{\ell}$. Note that the multiplication by the factor $\prod_{j=2}^{k+1} P_{N_{j}} u_{j}$ in (5.14) changes the time frequency at most by $O\left(N_{2}^{2}\right)$. Hence, $P_{R_{\ell}} P_{N_{1}} u_{1} \prod_{j=2}^{k+1} P_{N_{j}} u_{j}$ in (5.14) is localized at time frequency $M^{2} \ell^{2}+O\left(M^{2} \ell\right)=O\left(M^{2} \ell^{2}\right)$ for each $\ell$. Therefore, the sum in (5.14) is almost orthogonal and we have

$$
\left\|P_{C} P_{N_{1}} u_{1} \prod_{j=2}^{k+1} P_{N_{j}} u_{j}\right\|_{L_{t, \mathbf{x}}^{2}}^{2} \sim \sum_{\ell}\left\|P_{R_{\ell}} P_{N_{1}} u_{1} \prod_{j=2}^{k+1} P_{N_{j}} u_{j}\right\|_{L_{t, \mathbf{x}}^{2}}^{2} .
$$

With $d$ and $k$ as in (5.3), let $p_{d, k}=(d+2) k$. Then, by Lemma [5.9, we have

$$
\left\|P_{N} e^{-i t \Delta} \phi\right\|_{L_{t, \mathbf{x}}^{p_{d, k}}} \lesssim N^{s_{c}}\left\|P_{N} \phi\right\|_{L_{\mathbf{x}}^{2}}
$$

Now, choose $p$ such that

$$
\begin{aligned}
& \text { (i) } \frac{20}{3}<p<\frac{8 k}{k+1} \quad \text { when } d=2, \\
& \text { (ii) } \frac{16}{3}<p<\frac{20 k}{3 k+2} \quad \text { when } d=3 \\
& \text { (iii) } 4<p<\frac{4 k(d+2)}{d k+2} \quad \text { when } d \geq 4 .
\end{aligned}
$$


The existence of such $p$ is implied by (5.3). Moreover, the lower bound on $p$ guarantees that each $(d, p)$ is admissible, while the upper bound on $p$ guarantees that

$$
d-\frac{2(d+2)}{p}-s_{c}<0 \text {. }
$$

Let $q$ such that

$$
\frac{2}{p}+\frac{k-2}{p_{d, k}}+\frac{1}{q}=\frac{1}{2}
$$

Then, it follows from (5.17) that $(d, q)$ is also admissible. By Hölder's inequality and Lemmata 5.9 and 5.10 , we have

$$
\begin{aligned}
\| P_{R_{\ell}} P_{N_{1}} u_{1} & \prod_{j=2}^{k} P_{N_{j}} u_{j}\left\|_{L_{t, \mathbf{x}}^{2}} \lesssim\right\| P_{R_{\ell}} P_{N_{1}} u_{1}\left\|_{L^{p}}\right\| P_{N_{2}} u_{2}\left\|_{L^{p}} \prod_{j=3}^{k}\right\| P_{N_{j}} u_{j}\left\|_{L^{p}, k}\right\| P_{N_{k+1}} u_{k+1} \|_{L^{q}} \\
& \lesssim N_{2}^{d-\frac{2(d+2)}{p}-s_{c}} N_{k+1}^{\frac{d}{2}-\frac{d+2}{q}-s_{c}}\left(\frac{M}{N_{2}}\right)^{\delta}\left\|P_{R_{\ell}} P_{N_{1}} \phi_{1}\right\|_{L_{\mathbf{x}}^{2}} \prod_{j=2}^{k+1} N_{j}^{s_{c}}\left\|P_{N_{j}} \phi_{j}\right\|_{L_{\mathbf{x}}^{2}}, \quad(5.20)
\end{aligned}
$$

for some $\delta>0$. In view of (5.17) and (5.18), choose $p$ such that

$$
-d+\frac{2(d+2)}{p}+s_{c}=\delta .
$$

Moreover, from (5.18) and (5.19), we have

$$
\frac{d}{2}-\frac{d+2}{q}-s_{c}=-d+\frac{2(d+2)}{p}+s_{c}=\delta>0 .
$$

Then, noting $\frac{M}{N_{2}} \sim \frac{N_{2}}{N_{1}}+\frac{1}{N_{2}}$, it follows from (5.20) that

$$
\left\|P_{R_{\ell}} P_{N_{1}} u_{1} \prod_{j=2}^{k+1} P_{N_{j}} u_{j}\right\|_{L_{t, \mathbf{x}}^{2}} \lesssim\left(\frac{N_{k+1}}{N_{1}}+\frac{1}{N_{2}}\right)^{\delta}\left\|P_{R_{\ell}} P_{N_{1}} \phi_{1}\right\|_{L_{\mathbf{x}}^{2}} \prod_{j=2}^{k+1} N_{j}^{s_{c}}\left\|P_{N_{j}} \phi_{j}\right\|_{L_{\mathbf{x}}^{2}} .
$$

Finally, by summing up the squares in (5.15) with respect to $\ell$, we obtain (5.13) and hence (5.5). This completes the proof of Proposition 5.7.

5.3. Proof of Proposition 5.6. First, we state and prove an auxiliary lemma (Lemma 5.11), using Proposition [5.7. Let $\mathcal{C}_{N}, N \geq 1$, be the collection of cubes $C \subset \mathbb{Z}^{d}$ of side length $\sim N$ as before. Let $(d, p)$ be admissible in the sense of Definition 5.8. Then, it follows from Lemma 5.9 with Lemma 5.3 (i) that

$$
\left\|P_{C} e^{i t \Delta} \phi\right\|_{L_{t, \mathbf{x}}^{p}\left(I \times \mathbb{T}^{d}\right)} \lesssim N^{\frac{d}{2}-\frac{d+2}{p}}\left\|P_{C} \phi\right\|_{U_{\Delta}^{p} L_{\mathbf{x}}^{2}}
$$

for all $C \in \mathcal{C}_{N}$.

Lemma 5.11. Let $d$ and $k$ satisfy (5.3). Then, there exists $\delta^{\prime}>0$ such that

$$
\left\|\prod_{j=1}^{k+1} P_{N_{j}} u_{j}\right\|_{L_{t, \mathbf{x}}^{2}\left(I \times \mathbb{T}^{d}\right)} \lesssim\left(\frac{N_{k+1}}{N_{1}}+\frac{1}{N_{2}}\right)^{\delta^{\prime}}\left\|P_{N_{1}} u_{1}\right\|_{Y^{0}} \prod_{j=2}^{k+1}\left\|P_{N_{j}} u_{j}\right\|_{Y^{s_{c}}}
$$

for any interval $I \subset[0,1]$ and for all $N_{1} \geq N_{2} \geq \cdots \geq N_{k+1} \geq 1$. 
Proof. By almost orthogonality in spatial frequencies, it suffices to prove that there exists $\delta^{\prime}>0$ such that

$$
\left\|P_{C} P_{N_{1}} u_{1} \prod_{j=2}^{k+1} P_{N_{j}} u_{j}\right\|_{L_{t, \mathbf{x}}^{2}} \lesssim\left(\frac{N_{k+1}}{N_{1}}+\frac{1}{N_{2}}\right)^{\delta^{\prime}}\left\|P_{C} P_{N_{1}} u_{1}\right\|_{Y^{0}} \prod_{j=2}^{k+1} N_{j}^{s_{c}}\left\|P_{N_{j}} u_{j}\right\|_{Y^{0}}
$$

for all cubes $C \in \mathcal{C}_{N_{2}}$. Moreover, by the embedding (5.2), it suffices to prove (5.24), where the $Y^{0}$-norm is replaced by the $V_{\Delta}^{2} L^{2}$-norm. Furthermore, it suffices to prove that there exists $\lambda>0$ such that the following two estimates hold:

$$
\text { LHS of (15.24) } \lesssim\left(\frac{N_{k+1}}{N_{1}}+\frac{1}{N_{2}}\right)^{\delta}\left\|P_{C} P_{N_{1}} u_{1}\right\|_{U_{\Delta}^{2} L^{2}} \prod_{j=2}^{k+1} N_{j}^{s_{c}}\left\|P_{N_{j}} u_{j}\right\|_{U_{\Delta}^{2} L^{2}},
$$

and

$$
\text { LHS of }\left(\underline{5.24)} \lesssim\left(\frac{N_{k+1}}{N_{2}}\right)^{\delta}\left\|P_{C} P_{N_{1}} u_{1}\right\|_{U_{\Delta}^{p} L^{2}} \prod_{j=2}^{k+1} N_{j}^{s_{c}}\left\|P_{N_{j}} u_{j}\right\|_{U_{\Delta}^{p} L^{2}},\right.
$$

for some $p>2$. Indeed, if (5.25) and (5.26) hold, then it follows from Lemma 5.3 (ii) that (5.24) holds with $\delta^{\prime}<\delta$.

The first estimate (5.25) directly follows from Proposition 5.7 and Lemma 5.3 (i). Hence, it remains to prove the second estimate (5.26). Let $p, p_{d, k}$, and $q$ be as in the proof of Proposition 5.7. Then, by Hölder's inequality with (5.22), we have

$$
\begin{aligned}
\| P_{C} P_{N_{1}} u_{1} & \prod_{j=2}^{k} P_{N_{j}} u_{j}\left\|_{L_{t, \mathbf{x}}^{2}} \lesssim\right\| P_{C} P_{N_{1}} u_{1}\left\|_{L^{p}}\right\| P_{N_{2}} u_{2}\left\|_{L^{p}} \prod_{j=3}^{k}\right\| P_{N_{j}} u_{j}\left\|_{L^{p_{d, k}}}\right\| P_{N_{k+1}} u_{k+1} \|_{L^{q}} \\
& \lesssim N_{2}^{d-\frac{2(d+2)}{p}-s_{c}} N_{k+1}^{\frac{d}{2}-\frac{d+2}{q}-s_{c}}\left\|P_{C} P_{N_{1}} u_{1}\right\|_{U_{\Delta}^{p} L^{2}} \prod_{j=2}^{k+1} N_{j}^{s_{c}}\left\|P_{N_{j}} u_{j}\right\|_{U_{\Delta}^{q_{j}} L^{2}},
\end{aligned}
$$

where $q_{2}=p, q_{j}=p_{d, k}$ for $j=3, \ldots, k$, and $q_{k+1}=q$. From (5.17) and (5.19) with $p_{d, k}=(d+2) k$, we have $q>p_{d, k}>p>2$. Therefore, (5.26) follows from (5.27) with Remark 5.2 and (5.21).

We conclude this section by presenting the proof of Proposition 5.6.

Proof of Proposition 5.6. Let $I=[0, T)$. In the following, we prove

$$
\left\|\mathcal{I}\left(\mathbb{P}_{\leq N}\left(\prod_{j=1}^{2 k+1} u_{j}^{*}\right)\right)\right\|_{X^{s_{c}(I)}} \lesssim \sum_{j=1}^{2 k+1}\left(\left\|u_{j}\right\|_{X^{s}(I)} \prod_{\substack{\ell=1 \\ \ell \neq j}}^{2 k+1}\left\|u_{\ell}\right\|_{X^{s_{c}}(I)}\right)
$$

for all $N \geq 1$, where the implicit constant is independent of $N$. By Lemma 5.5, we have

$$
\left\|\mathcal{I}\left(\mathbb{P}_{\leq N}\left(\prod_{j=1}^{2 k+1} u_{j}^{*}\right)\right)\right\|_{X^{s_{c}(I)}} \leq \sup _{\substack{v \in Y^{-s}([0, T)) \\\|v\|_{Y^{-s}=1}}}\left|\int_{I \times \mathbb{T}^{d}} \prod_{j=1}^{2 k+1} u_{j}^{*}(\mathbf{x}, t) \mathbb{P}_{\leq N} \overline{v(\mathbf{x}, t)} d \mathbf{x} d t\right|
$$


Hence, with $u_{0}=\mathbb{P}_{\leq N} v$, it suffices to show that

$$
\left|\int_{I \times \mathbb{T}^{d}} \prod_{j=0}^{2 k+1} u_{j}^{*}(\mathbf{x}, t) d \mathbf{x} d t\right| \lesssim\left\|u_{0}\right\|_{Y^{-s}(I)} \sum_{j=1}^{2 k+1}\left(\left\|u_{j}\right\|_{X^{s}} \prod_{\substack{\ell=1 \\ \ell \neq j}}^{2 k+1}\left\|u_{\ell}\right\|_{X^{s_{c}}}\right) .
$$

Now, dyadically decompose $u_{j}^{*}=\sum_{N_{j} \geq 1} P_{N_{j}} u_{j}^{*}$. Without loss of generality, assume $N_{1} \geq N_{2} \geq \cdots \geq N_{2 k+1}$. Then, in order to have a non-trivial contribution on the left-hand side of (5.29), we must have $N_{1} \sim \max \left(N_{0}, N_{2}\right)$.

Case (i): $N_{0} \sim N_{1}$.

By Lemma 5.11, we have

$$
\begin{aligned}
& \left|\int_{I \times \mathbb{T}^{d}} \prod_{j=0}^{2 k+1} P_{N_{j}} u_{j}^{*}(\mathbf{x}, t) d \mathbf{x} d t\right| \leq\left\|\prod_{j=0}^{k} P_{N_{2 j}} u_{2 j}^{*}\right\|_{L_{t, \mathbf{x}}^{2}}\left\|\prod_{j=0}^{k} P_{N_{2 j+1}} u_{2 j+1}^{*}\right\|_{L_{t, \mathbf{x}}^{2}} \\
& \quad \lesssim\left(\frac{N_{2 k}}{N_{0}}+\frac{1}{N_{2}}\right)^{\delta^{\prime}}\left(\frac{N_{2 k+1}}{N_{1}}+\frac{1}{N_{3}}\right)^{\delta^{\prime}}\left\|P_{N_{0}} u_{0}\right\|_{Y^{-s}}\left\|P_{N_{1}} u_{1}\right\|_{Y^{s}} \prod_{j=2}^{2 k+1}\left\|P_{N_{j}} u_{j}\right\|_{Y^{s_{c}}} .
\end{aligned}
$$

Summing (5.30) over dyadic blocks $N_{0} \sim N_{1} \geq N_{2} \geq \cdots \geq N_{2 k+1}$ and by Cauchy-Schwarz inequality, we have

$$
\begin{aligned}
\text { LHS of (5.29) } & \lesssim \sum_{N_{0} \sim N_{1}}\left\|P_{N_{0}} u_{0}\right\|_{Y^{-s}}\left\|P_{N_{1}} u_{1}\right\|_{Y^{s}} \prod_{j=2}^{2 k+1}\left\|u_{j}\right\|_{Y^{s_{c}}} \\
& \lesssim\left\|u_{0}\right\|_{Y^{-s}}\left\|u_{1}\right\|_{Y^{s}} \prod_{j=2}^{2 k+1}\left\|u_{j}\right\|_{Y^{s_{c}}},
\end{aligned}
$$

yielding (5.29) in view of (5.2).

Case (ii): $N_{2} \sim N_{1} \gg N_{0}$.

By Lemma 5.11 with $N_{1} \sim N_{2}$, we have

$$
\begin{aligned}
\mid \int_{I \times \mathbb{T}^{d}} & \prod_{j=0}^{2 k+1} P_{N_{j}} u_{j}^{*}(\mathbf{x}, t) d \mathbf{x} d t \mid \\
& \lesssim\left(\frac{N_{0}}{N_{1}}\right)^{s+s_{c}}\left(\frac{N_{2 k+1}}{N_{1}}+\frac{1}{N_{3}}\right)^{\delta^{\prime}}\left\|P_{N_{0}} u_{0}\right\|_{Y^{0}}\left\|P_{N_{1}} u_{1}\right\|_{Y^{s}} \prod_{j=2}^{2 k+1}\left\|P_{N_{j}} u_{j}\right\|_{Y^{s_{c}}}
\end{aligned}
$$

Summing (5.31) over dyadic blocks $N_{0}\left(\ll N_{1}\right)$ and $N_{1} \sim N_{2} \geq N_{3} \geq \cdots \geq N_{2 k+1}$ and by Cauchy-Schwarz inequality, we have

$$
\begin{aligned}
\text { LHS of (5.29) } & \lesssim\left\|u_{0}\right\|_{Y^{-s}} \sum_{N_{1} \sim N_{2}}\left\|P_{N_{1}} u_{1}\right\|_{Y^{s}}\left\|P_{N_{2}} u_{2}\right\|_{Y^{s_{c}}} \prod_{j=3}^{2 k+1}\left\|u_{j}\right\|_{Y^{s_{c}}} \\
& \lesssim\left\|u_{0}\right\|_{Y^{-s}}\left\|u_{1}\right\|_{Y^{s}} \prod_{j=2}^{2 k+1}\left\|u_{j}\right\|_{Y^{s_{c}}} .
\end{aligned}
$$

This completes the proof of Proposition 5.6 . 


\section{Appendix A. On the Weyl sum estimate (2.11)}

In this appendix, we present a proof of (2.11). We decided to include the proof for the convenience of readers, in particular for those in PDEs. Since $I=I(\eta)$ is a compact interval and the integrand is periodic with period 1 , it suffices to show

$$
\int_{0}^{1}|F(t)|^{r} d t \sim N^{r-2}
$$

for $r>4$, where $F(t)$ is the Weyl sum defined by

$$
F(t)=\sum_{0 \leq n \leq N} e^{2 \pi i n^{2} t}
$$

For $a, q \in \mathbb{N}$ with $1 \leq a \leq q \leq N$ and $(a, q)=1$, define a major arc $\mathfrak{M}(q, a)$ by

$$
\mathfrak{M}(q, a)=\left\{t \in[0,1]:\left\|t-\frac{a}{q}\right\| \leq \frac{1}{100 N^{2}}\right\}
$$

where $\|x\|=\min _{n \in \mathbb{Z}}|x-n|$ denotes the distance of $x$ to the closest integer as before. Let $\mathfrak{M}=\bigcup_{a, q} \mathfrak{M}(q, a)$. Note that we have $\left\|t-\frac{a}{q}\right\| \leq \frac{1}{q^{2}}$ for $t \in \mathfrak{M}$. Then, by Weyl's inequality we have

$$
|F(t)| \lesssim \frac{N}{q^{\frac{1}{2}}}+N^{\frac{1}{2}}(\log q)^{\frac{1}{2}}+q^{\frac{1}{2}}(\log q)^{\frac{1}{2}}
$$

Hence, the contribution from the major arc $\mathfrak{M}$ is estimated by

$$
\int_{\mathfrak{M}}|F(t)|^{r} d t \lesssim \sum_{q=1}^{N} \sum_{\substack{a=1 \\(a, q)=1}}^{q} \frac{N^{r}}{q^{\frac{r}{2}}}(\log q)^{\frac{r}{2}} \frac{1}{N^{2}} \leq N^{r-2} \sum_{q=1}^{N} q^{1-\frac{r}{2}+} \lesssim N^{r-2},
$$

since $r>4$.

Remark A.1. Indeed, the contribution from the major arc $\mathfrak{M}$ provides the lower bound in (A.1). We only need to consider the contribution from $\mathfrak{M}(q, a)$ for odd $q \leq N^{\frac{1}{2}}$. Let $S(q, a)$ be the Gauss sum given by $S(q, a)=\sum_{n=1}^{q} e^{2 \pi i n^{2} \frac{a}{q}}$. We have $S(q, a)=\sqrt{q}$ for odd $q$. Now, suppose that $q$ is odd such that $1 \leq q \leq N^{\frac{1}{2}}$. Then, by Van der Corput's method [41] with $S(q, a)=\sqrt{q}$, one can show that $|F(t)| \gtrsim \frac{N}{q^{\frac{1}{2}}}$ for $t \in \mathfrak{M}(q, a)$. Noting that $\mathfrak{M}(q, a)$ are disjoint, we have

$$
\int_{\mathfrak{M}}|F(t)|^{r} d t \gtrsim \sum_{\substack{q=1 \\ q, \text { odd }}}^{N^{\frac{1}{2}}} \sum_{\substack{a=1 \\(a, q)=1}}^{q} \frac{N^{r}}{q^{\frac{r}{2}}} \frac{1}{N^{2}}=N^{r-2} \sum_{\substack{q=1 \\ q, \text { odd }}}^{N^{\frac{1}{2}}} \phi(q) q^{-\frac{r}{2}} \sim N^{r-2},
$$

where $\phi(q)$ denotes Euler's function. This shows the lower bound in (A.1).

Next, we estimate the contribution from the minor arc $\mathfrak{m}:=[0,1] \backslash \mathfrak{M}$. Fix small $\varepsilon>0$. Then, Dirichlet's theorem [40, Lemma 2.1] states that given $t \in[0,1]$, there exist integers $a, q$ with $1 \leq a \leq q \leq N^{2-\varepsilon}$ and $(a, q)=1$ such that $\left\|t-\frac{a}{q}\right\| \leq \frac{1}{q N^{2-\varepsilon}}$. Define $I(q)$ by

$$
I(q)=\bigcup_{\substack{a=1 \\(a, q)=1}}^{q} I(q, a)
$$


where $I(q, a)=\left\{t \in[0,1]:\left\|t-\frac{a}{q}\right\| \leq \frac{1}{q N^{2-\varepsilon}}\right\}$. Now, in view of (A.2), divide the minor arc $\mathfrak{m}$ into two pieces: $\mathfrak{m}=\mathfrak{m}_{1} \cup \mathfrak{m}_{2}$, where

$$
\mathfrak{m}_{1}=\mathfrak{m} \cap \bigcup_{1 \leq q \ll N^{\varepsilon}} I(q) \text { and } \quad \mathfrak{m}_{2}=\mathfrak{m} \cap \bigcup_{N<q \leq N^{2-\varepsilon}} I(q) .
$$

Let $t \in \mathfrak{m}_{2}$. From (A.3), we have $|F(t)| \lesssim N^{1-\frac{1}{2} \varepsilon}(\log N)^{\frac{1}{2}}$. Then, by Hua's inequality [40, Lemma 2.5], we have

$$
\begin{aligned}
\int_{\mathfrak{m}_{2}}|F(t)|^{r} d t & \leq\left(\sup _{t \in \mathfrak{m}_{2}}|F(t)|\right)^{r-4} \int_{0}^{1}|F(t)|^{4} d t \lesssim\left[N^{1-\frac{1}{2} \varepsilon}(\log N)^{\frac{1}{2}}\right]^{r-4} N^{2+} \\
& \leq N^{r-2} .
\end{aligned}
$$

Let $t \in \mathfrak{m}_{1}$, i.e. we have $\left\|t-\frac{a}{q}\right\| \leq \frac{1}{q N^{2-\varepsilon}}$ for some $q \ll N^{\varepsilon}$. Then, by Lemmata 2.7 and 2.8 in [40] with $|S(q, a)| \lesssim q^{\frac{1}{2}}$, we have

$$
|F(t)|=q^{-1} S(q, a) v\left(t-\frac{a}{q}\right)+O\left(N^{\frac{2}{200}}\right) \lesssim N^{1-\frac{\varepsilon}{2}},
$$

where $v$ is defined in [40, (2.9)]. Applying Hua's inequality as before, we have

$$
\int_{\mathfrak{m}_{1}}|F(t)|^{r} d t \leq\left(\sup _{t \in \mathfrak{m}_{1}}|F(t)|\right)^{r-4} \int_{0}^{1}|F(t)|^{4} d t \lesssim\left[N^{1-\frac{\varepsilon}{2}}\right]^{r-4} N^{2+} \leq N^{r-2} .
$$

This completes the proof of (A.1).

\section{Appendix B. On a partially irrational torus $\mathbb{T}^{2} \times \mathbb{T}_{\alpha_{3}}$}

In this appendix, we present a sketch of the proof of Theorem 1.6. Thus, we set $d=3$ and assume that (1.27) holds in the following. The main ingredient is an improvement of the Strichartz estimate on a partially irrational torus.

Proposition B.1. Let $d=3$ and $I$ be a compact interval in $\mathbb{R}$. Suppose that (1.27) holds. Then, the scaling-invariant Strichartz estimate (1.19) holds for $p>\frac{14}{3}$.

The proof of Proposition B.1 is based on the following level set estimates under the assumption (1.27).

Lemma B.2. Suppose that (1.27) holds. Given a compact interval $I \subset \mathbb{R}$ and $f$ as in (3.2), let $A_{\lambda}=A_{\lambda}(f)$ be the distribution function defined by (3.3).

(i) For any $\varepsilon>0$, we have

$$
\left|A_{\lambda}\right| \lesssim N^{\frac{1}{1+4 \varepsilon}} \lambda^{-4+\frac{8}{1+4 \varepsilon} \varepsilon}
$$

for $\lambda \gtrsim N^{1+\varepsilon}$.

(ii) Let $q>4$. Then, there exists small $\varepsilon>0$ such that

$$
\left|A_{\lambda}\right| \lesssim N^{\frac{3}{2} q-5} \lambda^{-q}
$$

for $\lambda \gtrsim N^{\frac{3}{2}-\varepsilon}$.

In (B.1) and (B.2), the implicit constants depend on $\varepsilon>0, q>4$, and $|I|$, but are independent of $f$. 
We first prove Proposition B.1 assuming Lemma B.2. Then, we sketch the proof of Theorem 1.6. We present the proof of Lemma B.2 at the end of this appendix.

Given $f$ as in (3.2), let $F(\mathbf{x}, t)=e^{-i t \Delta} f(\mathbf{x}, t)$. By Cauchy-Schwarz inequality, we have $\|F\|_{L_{t, \mathbf{x}}^{\infty}} \lesssim N^{\frac{3}{2}}$. Given $p>\frac{14}{3}$, let $q \in(4, p)$. Then, by Lemma B.2 and Theorem 1.1 (ii), we have

$$
\begin{aligned}
& \int_{I \times \mathbb{T}^{3}}|F(\mathbf{x}, t)|^{p} d \mathbf{x} d t
\end{aligned}
$$

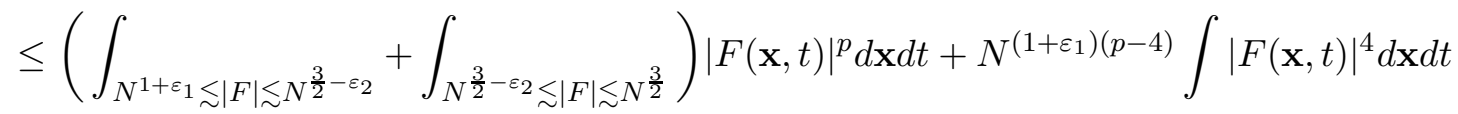

$$
\begin{aligned}
& \lesssim N^{1-\frac{4}{1+4 \varepsilon_{1}} \varepsilon_{1}} \int_{N^{1+\varepsilon_{1}}}^{N^{\frac{3}{2}-\varepsilon_{2}}} \lambda^{p-4+\frac{8}{1+4 \varepsilon_{1}} \varepsilon_{1}} d \lambda+N^{\frac{3}{2} q-5} \int_{N^{\frac{3}{2}-\varepsilon_{2}}}^{N^{\frac{3}{2}}} \lambda^{p-1-q} d \lambda+N^{\left(1+\varepsilon_{1}\right)(p-4)+\frac{4}{3}+} \\
& \lesssim N^{\frac{3}{2} p-5}
\end{aligned}
$$

for sufficiently small $\varepsilon_{1}, \varepsilon_{2}>0$. Here, the condition $p>\frac{14}{3}$ is needed in the last inequality. This proves Proposition B.1.

Next, we briefly discuss how Theorem 1.6 follows from Proposition B.1. As in Section 5. the goal is to prove the multilinear estimate (5.4) in Proposition 5.6 for $d=3$ and $k=2$ (with $s_{c}=1$ ) under the assumption (1.27). In view of the argument in Subsection 5.3 (which holds without any change even in this case), it suffices to prove the multilinear Strichartz estimate (5.5) in Proposition 5.7 for $d=3$ and $k=2$ (with $s_{c}=1$ ). By repeating the proof of Lemma 5.10 with Proposition B.1, we see that (5.11) holds for $p>\frac{14}{3}$ with $\delta \in\left(0, \frac{1}{2}-\frac{7}{3 p}\right)$. In the proof of Proposition 5.7, the only change appears in (5.17) and we can choose $p$ such that

$$
\frac{14}{3}<p<\frac{20 k}{3 k+2}
$$

in this case. In particular, we can set $k=2$ by choosing $p \in\left(\frac{14}{3}, 5\right)$. The rest of the argument follows exactly as in Section 5 .

In the remaining part of the paper, we present the proof of Lemma B.2.

Proof of Lemma B.2. The proof follows the proof of Proposition 3.1 with small modifications. In the following, we only point out these modifications. Given an interval $I \subset \mathbb{R}$, assume that $I$ is centered at 0 .

(i) With $Q(\mathbf{n})$ in (1.27), define $\mathbf{R}$ as in (2.19). Then, we have $\mathbf{R}(\mathbf{x}, t)=\prod_{j=1}^{2} R_{1}\left(x_{j}, t\right)$. $R_{\theta_{3}}\left(x_{3}, t\right)$, where $R_{\theta}$ is defined in (2.20). We also define $\mathbf{R}_{1}$ and $\mathbf{R}_{2}$ as in (3.10). Then, by (3.11), we have

$$
\left\|\mathbf{R}_{1}\right\|_{L_{t, \mathbf{x}}^{\infty}} \lesssim \min \left(N M \log M, N^{3}\right) .
$$

Proceeding as in (3.18) with (B.3) and (3.15), we have

$$
\begin{aligned}
\lambda^{2}\left|A_{\lambda}\right|^{2} & \leq\left\|\mathbf{R}_{1}\right\|_{L_{t, \mathbf{x}}^{\infty}}\left\|\Theta_{\lambda}\right\|_{L_{t, \mathbf{x}}^{1}}^{2}+\left\|\widehat{\mathbf{R}}_{2}\right\|_{L_{\tau}^{\infty} \ell_{\mathbf{n}}^{\infty}}\left\|\Theta_{\lambda}\right\|_{L_{t, \mathbf{x}}^{2}}^{2} \\
& \leq C_{1} N M^{1+\varepsilon_{1}}\left|A_{\lambda}\right|^{2}+M^{-1+\varepsilon_{2}}\left|A_{\lambda}\right|
\end{aligned}
$$


for small $\varepsilon_{1}, \varepsilon_{2}>0$. Choose $M \geq N$ such that $N M^{1+\varepsilon_{1}} \sim \lambda^{2}$. This condition with $M \geq N$ implies that $\lambda \gtrsim N^{1+\frac{\varepsilon_{1}}{2}}$. Then, (‥4) yields

$$
\left|A_{\lambda}\right| \lesssim\left(\frac{N}{\lambda^{2}}\right)^{\frac{1-\varepsilon_{2}}{1+\varepsilon_{1}}} \lambda^{-2} \lesssim N^{\frac{1}{1+2 \varepsilon_{1}}} \lambda^{-4+\frac{4}{1+2 \varepsilon_{1}} \varepsilon_{1}}
$$

by setting $\varepsilon_{2}=\varepsilon_{2}\left(\varepsilon_{1}\right)$ such that $\frac{1-\varepsilon_{2}}{1+\varepsilon_{1}}=\frac{1}{1+2 \varepsilon_{1}}$. This proves (B.1) with $\varepsilon=\frac{\varepsilon_{1}}{2}$.

(ii) Define $\mathbf{K}$ by

$$
\mathbf{K}(\mathbf{x}, t)=\chi(t) K\left(x_{1}, t\right) K\left(x_{2}, t\right) \sum_{\left|n_{3}\right| \leq N} e^{2 \pi i\left(n_{3} x_{3}+\theta_{3} n_{3}^{2} t\right)}
$$

where $K(x, t)$ is as in (3.20). Let $\Lambda_{M, s}$ be as in (3.31). Then, from Lemma 3.4, (3.26), and (3.29) with $M \leq 2^{s} \leq N$, we have

$$
\left|\Lambda_{M, s}(\mathbf{x}, t)\right| \lesssim N\left(\frac{N}{M^{\frac{1}{2}}\left(1+\left(2^{-s} N\right)^{\frac{1}{2}}\right)}\right)^{2}+\frac{M^{2}}{2^{s} N} N^{2} \lesssim \frac{2^{s} N^{2}}{M} .
$$

Hence, from (B.6), we have

$$
\left\|f * \Lambda_{M, s}\right\|_{L^{\infty}\left(I \times \mathbb{T}^{d}\right)} \lesssim \frac{2^{s} N^{2}}{M}\|f\|_{L^{1}\left(I \times \mathbb{T}^{d}\right)} .
$$

Also, with $\Lambda$ as in (3.46) and $\mathbf{K}$ as in (B.5), it follows from (3.26) and (3.29) with $N_{1}=\frac{1}{100} N$ that

$$
\|\mathbf{K}-\Lambda\|_{L^{\infty}\left(I \times \mathbb{T}^{d}\right)} \lesssim N^{2}
$$

Let $p$ be as in (3.50). In the following, we consider the case $\theta \geq \frac{1}{3}$. With $M_{1}$ and $M_{2}$ as in (3.56), write $\Lambda=\Lambda_{1}+\Lambda_{2}+\Lambda_{3}$ as before. See (3.57).

By interpolating (B.7) and (3.39) and summing over dyadic $M \leq M_{1}$ and $2^{s} \leq N$, we have

$$
\left\|f * \Lambda_{1}\right\|_{L^{p^{\prime}}\left(I \times \mathbb{T}^{d}\right)} \lesssim M_{1}^{-1+(3+) \theta} N^{3-5 \theta}\|f\|_{L^{p}\left(I \times \mathbb{T}^{d}\right)}
$$

since $\theta \in\left[\frac{1}{3}, \frac{1}{2}\right)$.

Next, choose $D \sim M^{\alpha}$ for some small $\alpha=\alpha(\theta)>0$, and set $\beta \ll 1$ and $B \gg 1$ such that

$$
\sigma:=-3-\beta+\alpha B->0 \text {. }
$$

Then, by interpolating (B.7) and (3.45) and summing over dyadic $M \in\left(M_{1}, M_{2}\right]$ and $2^{s} \leq N$, we have

$$
\begin{aligned}
\left\|f * \Lambda_{2}\right\|_{L^{p^{\prime}}\left(I \times \mathbb{T}^{d}\right)} & \lesssim N^{3-5 \theta}\|f\|_{L^{p}\left(I \times \mathbb{T}^{d}\right)} \\
& +\left(M_{1}^{-1-\sigma \theta} N^{3-\frac{5}{2} \theta}+M_{2}^{-1+(3+B+) \theta} N^{3-\frac{7}{2} \theta}\right)\|f\|_{L^{1}\left(I \times \mathbb{T}^{d}\right)},
\end{aligned}
$$

as long as $\theta<\frac{1}{2+\alpha+}$. This can be guaranteed by choosing $\alpha=\alpha(\theta)>0$ sufficiently small for given $\theta<\frac{1}{2}$.

Lastly, by interpolating (B.7) and (3.38) and summing over $M \geq M_{2} \sim N^{\delta_{2}}$ and $s$ with $2^{s} \leq N$, we have

$$
\left\|f * \Lambda_{3}\right\|_{L^{p^{\prime}}\left(I \times \mathbb{T}^{d}\right)} \lesssim N^{3-5 \theta}\|f\|_{L^{p}\left(I \times \mathbb{T}^{d}\right)}
$$

as long as $\theta<\frac{1}{2}$. 
Now, we are ready to prove the level set estimate (B.2) for $q>4$. Then, proceeding as before with (B.8), (B.9), (B.10), and (B.11), we have

$$
\begin{aligned}
\lambda^{2}\left|A_{\lambda}\right|^{2} & \leq\left|\left\langle(\mathbf{K}-\Lambda) * \Theta_{\lambda}, \Theta_{\lambda}\right\rangle\right|+\left|\left\langle\Lambda * \Theta_{\lambda}, \Theta_{\lambda}\right\rangle\right| \\
& \lesssim N^{2}\left|A_{\lambda}\right|^{2}+M_{1}^{-1+(3+) \theta} N^{3-5 \theta}\left|A_{\lambda}\right|^{\frac{2}{p}} \\
& \quad+M_{1}^{-1-\sigma \theta} N^{3-\frac{5}{2} \theta}\left|A_{\lambda}\right|^{1+\frac{1}{p}}+M_{2}^{-1+(3+B+) \theta} N^{3-\frac{7}{2} \theta}\left|A_{\lambda}\right|^{1+\frac{1}{p}}
\end{aligned}
$$

Since $\lambda \gg N$, (B.12) reduces to

$$
\begin{aligned}
\lambda^{2}\left|A_{\lambda}\right|^{2} \lesssim & M_{1}^{-1+(3+) \theta} N^{3-5 \theta}\left|A_{\lambda}\right|^{\frac{2}{p}}+M_{1}^{-1-\sigma \theta} N^{3-\frac{5}{2} \theta}\left|A_{\lambda}\right|^{1+\frac{1}{p}} \\
& \quad+M_{2}^{-1+(3+B+) \theta} N^{3-\frac{7}{2} \theta}\left|A_{\lambda}\right|^{1+\frac{1}{p}} \\
= & \mathrm{I}+\mathrm{II}+\mathrm{III} .
\end{aligned}
$$

First, suppose that $\lambda^{2}\left|A_{\lambda}\right|^{2} \lesssim$ I holds. With $p^{\prime} \theta=2$ and (3.56), we have

$$
\left|A_{\lambda}\right| \lesssim\left(\frac{N^{\frac{3}{2}}}{\lambda}\right)^{\left(-\frac{p^{\prime}}{2}+3+\right) \delta_{1}} N^{\frac{3}{2} p^{\prime}-5} \lambda^{-p^{\prime}} \lesssim N^{\frac{3}{2} q-5} \lambda^{-q}
$$

for $q>p^{\prime}$ by choosing $\delta_{1}=\delta_{1}\left(q, p^{\prime}\right)$ sufficiently small. Next, suppose that $\lambda^{2}\left|A_{\lambda}\right|^{2} \lesssim I$ holds. Then, from (3.56), we have

$$
\left|A_{\lambda}\right| \lesssim N^{\frac{3}{2} p^{\prime}-5} \lambda^{-p^{\prime}}\left(N^{\frac{3}{2} p^{\prime}} \lambda^{-p^{\prime}} M_{1}^{-p^{\prime}-2 \sigma}\right) \lesssim N^{\frac{3}{2} p^{\prime}-5} \lambda^{-p^{\prime}},
$$

by making $\sigma=\sigma\left(p^{\prime}, \delta_{1}\right)$ in (3.60) (and hence $B=B\left(p^{\prime}, \delta_{1}\right)$ ) sufficiently large. Lastly, suppose that $\lambda^{2}\left|A_{\lambda}\right|^{2} \lesssim$ III holds. By $\lambda \gtrsim N^{\frac{3}{2}-\varepsilon}$ and (3.56), we have

$$
\left|A_{\lambda}\right| \lesssim N^{\frac{3}{2} p^{\prime}-5} \lambda^{-p^{\prime}} N^{-2+\varepsilon p^{\prime}+\left(-p^{\prime}+6+2 B+\right) \delta_{2}} \lesssim N^{\frac{3}{2} p^{\prime}-5} \lambda^{-p^{\prime}}
$$

as long as we have $\varepsilon p^{\prime} \leq 1$ and $\delta_{2}=\delta_{2}\left(p^{\prime}, B\right)$ is sufficiently small.

Finally, given $q>4$, we choose $\theta<\frac{1}{2}$ such that $q>p^{\prime}=\frac{2}{\theta}>4$. Then, (B.2) follows from (B.13), (B.14), (B.15), and (B.16) with $\lambda \leq N^{\frac{3}{2}}$.

\section{REFERENCES}

[1] J. Bennett, A. Carbery, T. Tao, On the multilinear restriction and Kakeya conjectures, Acta Math. 196 (2006), no. 2, 261-302.

[2] J. Bourgain, On $\Lambda(p)$-subsets of squares, Israel J. Math. 67 (1989), no. 3, 291-311.

[3] J. Bourgain, Fourier transform restriction phenomena for certain lattice subsets and applications to nonlinear evolution equations. I. Schrödinger equations, Geom. Funct. Anal. 3 (1993), 107-156.

[4] J. Bourgain, Exponential sums and nonlinear Schrödinger equations, Geom. Funct. Anal. 3 (1993), $157-178$.

[5] J. Bourgain, Nonlinear Schrödinger equations, Hyperbolic equations and frequency interactions (Park City, UT, 1995), 3-157, IAS/Park City Math. Ser., 5, Amer. Math. Soc., Providence, RI, 1999.

[6] J. Bourgain, On Strichartz's inequalities and the nonlinear Schrödinger equation on irrational tori, Mathematical aspects of nonlinear dispersive equations, 1-20, Ann. of Math. Stud., 163, Princeton Univ. Press, Princeton, NJ, 2007.

[7] J. Bourgain, Moment inequalities for trigonometric polynomials with spectrum in curved hypersurfaces, Israel J. Math. 193 (2013), no. 1, 441-458.

[8] J. Bourgain, L. Guth, Bounds on oscillatory integral operators based on multilinear estimates, Geom. Funct. Anal. 21 (2011), no. 6, 1239-1295.

[9] N. Burq, P. Gérard, N. Tzvetkov, Strichartz inequalities and the nonlinear Schrödinger equation on compact manifolds, Amer. J. Math. 126 (2004), no. 3, 569-605. 
[10] N. Burq, P. Gérard, N. Tzvetkov, Bilinear eigenfunction estimates and the nonlinear Schrödinger equation on surfaces, Invent. Math. 159 (2005), no. 1, 187-223.

[11] F. Catoire, W.-M. Wang, Bounds on Sobolev norms for the defocusing nonlinear Schrödinger equation on general flat tori, Commun. Pure Appl. Anal. 9 (2010), 483-491.

[12] T. Cazenave, Semilinear Schrödinger equations, Courant Lecture Notes in Mathematics, 10. New York University, Courant Institute of Mathematical Sciences, New York; American Mathematical Society, Providence, RI, 2003. xiv +323 pp.

[13] T. Cazenave, F. Weissler, The Cauchy problem for the critical nonlinear Schrödinger equation in $H^{s}$, Nonlinear Anal. 14 (1990), no. 10, 807-836.

[14] C. Demeter, Incidence theory and restriction estimates, arXiv:1401.1873 [math.CA].

[15] S. Demirbas, Local well-posedness for 2-d Schrödinger equation on irrational tori and bounds on Sobolev norms, arXiv:1307.0051 [math.AP].

[16] J. Ginibre, G. Velo, On the global Cauchy problem for some nonlinear Schrödinger equations, Ann. Inst. H. Poincaré Anal. Non Linéaire 1 (1984), no. 4, 309-323.

[17] J. Ginibre, G. Velo, Smoothing properties and retarded estimates for some dispersive evolution equations. Comm. Math. Phys. 144 (1992), no. 1, 163-188.

[18] M. Hadac, S. Herr, H. Koch, Well-posedness and scattering for the KP-II equation in a critical space, Ann. Inst. H. Poincaré Anal. Non Linéaire 26 (2009), no. 3, 917-941. Erratum to "Well-posedness and scattering for the KP-II equation in a critical space", Ann. Inst. H. Poincaré Anal. Non Linéaire 27 (2010), no. 3, 971-972.

[19] G. Hardy, E. Wright, An introduction to the theory of numbers, Fifth edition. The Clarendon Press, Oxford University Press, New York, 1979. xvi+426 pp.

[20] S. Herr. The quintic nonlinear Schrödinger equation on three-dimensional Zoll manifolds, Amer. J. Math. 135 (2013), no. 5, 1271-1290.

[21] S. Herr, D. Tataru, N. Tzvetkov, Global well-posedness of the energy critical Nonlinear Schrödinger equation with small initial data in $H^{1}\left(\mathbb{T}^{3}\right)$, Duke Math. J., 159 (2011) 329-349.

[22] S. Herr, D. Tataru, N. Tzvetkov, Strichartz estimates for partially periodic solutions to Schrödinger equations in $4 d$ and applications, J. Reine Angew. Math. doi 10.1515/crelle-2012-0013.

[23] Y. Hu, X. Li, Discrete Fourier restriction associated with Schrödinger Equations, arXiv:1108.5164v1.

[24] M. Huxley, Exponential sums and lattice points. III, Proc. London Math. Soc. (3) 87 (2003), no. 3, 591-609.

[25] H. Iwaniec, E. Kowalski, Analytic number theory, American Mathematical Society Colloquium Publications, 53. American Mathematical Society, Providence, RI, 2004. xii+615 pp.

[26] A. Ionescu, B. Pausader, The energy-critical defocusing NLS on $\mathbb{T}^{3}$, Duke Math. J. 161 (2012), no. 8, 1581-1612.

[27] V. Jarník, Über die Gitterpunkte auf konvexen Kurven, (German) Math. Z. 24 (1926), no. 1, 500-518.

[28] T. Kato, On nonlinear Schrödinger equations, Ann. Inst. H. Poincaré Phys. Théor. 46 (1987), no. 1, 113-129.

[29] M. Keel, T. Tao, Endpoint Strichartz estimates. Amer. J. Math. 120 (1998), no. 5, 955-980.

[30] H. Koch, D. Tataru, A priori bounds for the 1D cubic NLS in negative Sobolev spaces, Int. Math. Res. Not. (2007), no. 16, Art. ID rnm053, 36 pp.

[31] H. Montgomery, Ten lectures on the interface between analytic number theory and harmonic analysis, CBMS Regional Conference Series in Mathematics, 84. Published for the Conference Board of the Mathematical Sciences, Washington, DC; by the American Mathematical Society, Providence, RI, 1994. $x i v+220$ pp.

[32] B. Pausader, N. Tzvetkov, X. Wang, Global regularity for the energy-critical NLS on $\mathbb{S}^{3}$, arXiv:1210.3842 [mathAP], to appear in Ann. Inst. H. Poincaré Anal. Non Linéaire.

[33] N. Rogovskaya, An asymptotic formula for the number of solutions of a system of equations, (Russian) Diophantine approximations, Part II (Russian), 78-84, Moskov. Gos. Univ., Moscow, 1986.

[34] R. S. Strichartz, Restrictions of Fourier transforms to quadratic surfaces and decay of solutions of wave equations. Duke Math. J. 44 (1977), no. 3, 705-714.

[35] N. Strunk, Strichartz estimates for Schrödinger equations on irrational tori in two and three dimensions arXiv:1401.6080 [math.AP].

[36] C. Sulem, P.L. Sulem, The nonlinear Schrödinger equation. Self-focusing and wave collapse, Applied Mathematical Sciences, 139. Springer-Verlag, New York, 1999. xvi+350 pp. 
Z. GUO, T. OH, AND Y. WANG

[37] H. Takaoka, N. Tzvetkov, On $2 D$ nonlinear Schrödinger equations with data on $\mathbb{R} \times \mathbb{T}$, J. Funct. Anal. 182 (2001), no. 2, 427-442.

[38] T. Tao, Nonlinear dispersive equations. Local and global analysis, CBMS Regional Conference Series in Mathematics, 106. Published for the Conference Board of the Mathematical Sciences, Washington, DC; by the American Mathematical Society, Providence, RI, 2006. xvi+373 pp.

[39] Y. Tsutsumi, $L^{2}$-solutions for nonlinear Schrödinger equations and nonlinear groups, Funkcial. Ekvac. 30 (1987), no. 1, 115-125.

[40] R. Vaughan, The Hardy-Littlewood method. Second edition, Cambridge Tracts in Mathematics, 125. Cambridge University Press, Cambridge, 1997. xiv +232 pp.

[41] I.M. Vinogradov, The method of trigonometrical sums in the theory of numbers, Translated from the Russian, revised and annotated by K. F. Roth and Anne Davenport. Reprint of the 1954 translation. Dover Publications, Inc., Mineola, NY, 2004. x+180 pp.

[42] Y. Wang, Periodic nonlinear Schrödinger equation in critical $H^{s}\left(\mathbb{T}^{n}\right)$ spaces, SIAM J. Math. Anal. 45 (2013), no. 3, 1691-1703.

[43] T. Wolff, Lectures on harmonic analysis, University Lecture Series, 29. American Mathematical Society, Providence, RI, 2003. x+137 pp.

[44] K. Yajima, Existence of solutions for Schrödinger evolution equations. Comm. Math. Phys. 110 (1987), no. 3, 415-426.

Zihua Guo, School of Mathematical Sciences, Peking University, Beijing 100871, China

E-mail address: zihuaguo@math.pku.edu.cn

Tadahiro Oh, School of Mathematics, The University of Edinburgh, and The Maxwell Institute for the Mathematical Sciences, James Clerk Maxwell Building, The King's Buildings, Mayfield Road, Edinburgh, EH9 3JZ, Scotland, and Department of Mathematics, Princeton University, Fine Hall, Washington Rd., Princeton, NJ 08544-1000, USA

E-mail address: hiro.oh@ed.ac.uk

Yuzhao Wang, Department of Mathematics and Physics, North China Electric Power UniVERsity, BeiJing 102206, China

Current address: Department of Mathematics and Statistics, Memorial University, St. John's, NL A1C 5S7, Canada

E-mail address: wangyuzhao2008@gmail.com 